\title{
La botanique dans un contexte local: les jardins de Florence à l'époque des grands-ducs (1569-1859)
}

René Sigrist et Sonia Zanier*

\section{Summary}

This article describes the social and institutional conditions of the practice of botany in early modern Florence. This practice started with the study of medical plants in hospital and university contexts, with the passion of the Medicis for gardens, and the interest of the Vallombrosian monks for cryptogams. During the XVIII th century, science of plants focused on classification (morphology), pharmacology (materia medica) and vegetable physiology, but included also the inventory of Tuscan flora and agronomy. These diverging aims created tensions within the nascent community of botanists, crystallizing around the management of gardens and the choice of classification systems. After 1770, a more scientific approach of botany was made possible by the rise of experimental practices and the development of chemistry. Yet, a true professionalization of research did not occur before the political unification of Italy, when the management of institutions and the recruitment of botanists were assumed by a central Ministry of education, instead of being dependent on princely favors and patrician connections.

Keywords: botany, Florence, $18^{\text {th }}$ century, gardens, agronomy

* Cette recherche, menée pour l'essentiel à l'Institut des Sciences Sociales de l'Université de Lausanne, a été financée par le Fonds National Suisse de la recherche scientifique, subsides no 100.011_137.579 et 100.011_166.345. Les auteurs tiennent à témoigner leur gratitude envers Emma Spary pour ses suggestions bibliographiques et interprétatives, ainsi que pour ses nombreuses remarques. Les erreurs qui peuvent subsister dans cet article demeurent néanmoins de leur seule responsabilité.

René Sigrist, Institut des Sciences Sociales, Faculté des sciences sociales et politiques, Université de Lausanne, CH-1015 Lausanne (sigrist.rene@bluewin.ch); Sonia Zahier, Via Sottomonte 4, I-33020 Sutrio (UD) (sonia.zahier@yahoo.it) 


\section{Résumé}

Cet article traite des conditions institutionnelles sociales de la pratique de la botanique dans la Florence des grands-ducs de Toscane (1569-1859). Cette pratique fut marquée à l'origine par l'étude des plantes médicinales dans un contexte hospitalier et universitaire, par la passion des Médicis pour les jardins et par l'intérêt des religieux de Vallombrosa pour les plantes cryptogames. Au XVIII siècle, les études botaniques se focalisent sur la classification (morphologie), la pharmacologie (materia medica) et la physiologie végétale, mais incluent aussi l'inventaire de la flore toscane, ainsi que l'agronomie. Ces multiples objectifs créent au sein de la communauté naissante des botanistes des tensions relatives à la gestion des différents jardins botaniques et au choix des systèmes de classification. A partir des années 1770, l'essor des pratiques expérimentales et le développement de la chimie accélèrent l'évolution vers une étude plus scientifique des végétaux. Celle-ci n'aboutit pourtant à une vraie professionnalisation de la recherche qu'après l'unification italienne, lorsque le sort des institutions et le choix de leurs titulaires dépendra d'un ministère central de l'instruction publique, et non plus des faveurs princières ou patriciennes.

Mots-clés: botanique, Florence, XVIIIe siècle, jardins, agronomie

Comme d'autres institutions scientifiques, les jardins botaniques délimitent des espaces consacrés à la recherche. Ils sont en principe destinés à mettre celle-ci à l'abri du monde extérieur en la préservant de toute forme de contamination sociale, politique, économique ou religieuse. Bien des historiens ont cependant démontré le caractère très relatif de cette préservation, ne serait-ce que parce que la définition même des fonctions d'une institution de recherche fait nécessairement l'objet de tractations sociales entre les différents acteurs impliqués, qu'ils soient chercheurs, administrateurs, mécènes ou encore destinataires du savoir produit. L'histoire des jardins botaniques de Florence au cours du XVIIIe siècle, ou même de toute la période dite moderne, peut servir à illustrer ce point. Elle vient compléter, par ses variations propres, une démonstration déjà apportée par des auteurs comme Emma Spary à propos du jardin des plantes de Paris ou comme Richard Drayton à propos du jardin de Kew. ${ }^{1}$ Nous y voyons que la science des plantes subit l'influence des contextes dans lesquelles elle s'insère et possède de ce fait une dimension locale: elle a donc une géo-

1 Spary 2000; Drayton 1993. 
graphie, en dépit des perspectives universelles de la recherche. Le cas de Florence confirme aussi que ces ancrages locaux évoluent au fil du temps. Les fonctions des établissements botaniques y sont en effet continuellement renégociées et redéfinies selon des rapports de force changeants.

La fondation en 1716 de la Società botanica pourrait constituer un point de épart commode pour un propos centré sur l'histoire sociale de la botanique à Florence. Ce choix ferait pourtant table rase de tout un passé, dont cette fondation fut en partie tributaire. Il faut savoir en effet que c'est dans la seconde moitié du XVI ${ }^{\mathrm{e}}$ siècle qu'ont été définies les règles et les programmes de l'enseignement de la botanique médicale, règles qui évoluèrent assez peu jusqu'à la fin du XVIII ${ }^{\mathrm{e}}$ siècle. ${ }^{2}$ L'histoire de la botanique florentine commence donc à la Renaissance, voire plus tôt. Il est en effet possible que les cours de médecine, dispensés depuis la fin du XIII ${ }^{e}$ siècle à l'hôpital de Santa Maria Nuova et organisés en un Studium generale universitaire depuis 1321, aient nécessité un jardin de plantes médicinales, ou à tout le moins un potager pour les malades. ${ }^{3}$ Quoi qu'il en soit, ce n'est qu'à partir de 1450 que ce premier jardin de plantes médicinales est attesté. ${ }^{4} \mathrm{Sa}$ dénomination de Giardino dei Semplici, que nous allons retrouver par la suite appliquée à d'autre jardins, en marque bien la destination première. La continuité historique de ce jardin, tout au long des deux siècles qui suivent, n'est pas absolument certaine en raison des multiples réorganisations et transferts répétés auxquels fut soumis le Studium generale. En 1473, Lorenzo de Médicis inaugura ainsi une longue période de conflits entre Florence et Pise en décidant que l'Université de la République serait transférée d'une ville à l'autre. La permanence du jardin de Santa Maria Nuova, dit aussi de Sant'Egidio, ne peut ainsi être assurée qu'à partir de 1650, date de la publication d'un catalogue de plantes rédigé par le médecin Giuseppe Baldi, qui en assuma la direction pendant trente-six ans. ${ }^{5}$ Entretemps, la botanique florentine s'était développée sur d'autres théâtres. ${ }^{6}$

L'un de ces théâtres était le Giardino botanico di San Marco, situé près du couvent du même nom, jardin tantôt désigné comme Giardino delle Stalle, tantôt comme Giardino dei Semplici, ce qui devait créer de fréquentes confusions, mais qui s'explique par le statut de la botanique à l'époque. ${ }^{7} \mathrm{Ce}$ jardin

2 Bellorini 2016, 57.

3 Coturri 1964; Lucarelle 1986; Palagi 1988; Mannelli-Poggioli (éd.) 2000.

4 Corti/Maugini/Lippini 1986, 746.

5 Mattirolo 1899, 23.

6 D'autres hôpitaux, comme celui des Innocents de Florence, ont également disposé d'un jardin de plantes médicinales à un moment ou à un autre de leur histoire.

7 Les termes de botanique et de botaniste n'apparaîtront qu'à la fin du XVII siècle, pour devenir d'usage courant au XVIII ${ }^{\mathrm{e}}$. Jusque-là, on parle de «res herbaria» pour la science des plantes, de «materia medica» ou de «lectura de simplicibus» (ou encore de «lectura 
fut officiellement créé le $1^{\text {er }}$ décembre 1545 , date de l'achat du terrain aux religieuses dominicaines, puis réalisé d'après des plans de l'architecte Niccolò Tribolo (fig. 1), qui conçut également, à peu près à la même époque, le jardin d'agrément de Boboli selon les indications du grand-duc Cosimo I ${ }^{\mathrm{Ie}}$ et de son épouse Eléonore de Tolède. Le véritable instigateur de cette création était Luca Ghini, qui avait été appelé l'année précédente à Pise pour y enseigner la materia medica, c'est-à-dire l'étude des principes médicamenteux tirés des plantes. La création d'un «jardin des simples» à Pise avait été une condition de son transfert depuis l'Université de Bologne. Par ricochet, elle avait entraîné la fondation d'un autre jardin à Florence, l'éternelle rivale de Pise. ${ }^{8}$ Ce jardin de San Marco, dont le premier directeur fut vraisemblablement Niccolò Gaddi (1499-1552), ${ }^{9}$ paraissait destiné aux étudiants du Studio Fiorentino, ainsi qu'au personnel des hôpitaux de la ville, auquel elle donnait les moyens de mieux se familiariser avec l'utilisation des plantes médicinales. ${ }^{10}$ Il semble pourtant que sa fonction première était de permettre aux étudiants de Pise, qui rentraient à Florence pendant les vacances d'été (vacances que Ghini passait lui-même à Bologne), d'y continuer leurs études sur les plantes. Ce lien organique est d'autant plus évident que Ghini fut chargé des plantations du jardin de Florence aussi bien que de celui de Pise. En outre, plusieurs directeurs devaient diriger conjointement les deux établissements, comme ce fut le cas de Giuseppe Casabona dans les années $1590 .{ }^{11}$

\section{L'héritage de la botanique renaissante}

Dès le milieu du XVI ${ }^{\mathrm{e}}$ siècle, l'Université de Pise joua, grâce à l'appui de Cosimo I ${ }^{\mathrm{er}}$, puis de ses successeurs François $\mathrm{I}^{\mathrm{er}}$ et Ferdinand $\mathrm{I}^{\mathrm{er}}$, un rôle dans la renaissance de la botanique médicale et dans son implantation en milieu universitaire. Cette université, remise sur pied en 1543-1544 après une longue période d'inactivité, était d'ailleurs une pièce essentielle de la politique culturelle de Cosimo de Médicis, qui avait été élu dès 1537 à la tête de

simplicium») pour la science enseignée dans une «cattedra dei semplici» par un «lector simplicium». Les simples étaient en effet des drogues faite d'un seul composant, généralement une plante, mais parfois aussi une substance animale ou minérale. Par conséquent un jardin où poussaient des plantes médicinales devenait un «orto» ou un «giardino dei semplici».

8 Le jardin de Pise était déjà en fonctions en juin 1545, lorsque fut prise la décision de créer celui de Padoue. Il précédait donc aussi celui de Florence de quelques mois (Engelhardt 2011, 67-68).

9 Patricien florentin, Gaddi était un grand voyageur, passionné de floriculture et d'horticulture, qui possédait lui-même un jardin avec nombre de plantes exotiques.

10 Corti/Maugini/Lippini 1986, 750; Luzzi/Fabbri 1993, 57.

11 Bellorini 2016, 93. Curieusement, c'est le nom de Giuseppe Benincasa.(c. 1500-1595) qui apparaît chez Engelhardt 2011, 67. 
la République de Florence en tant que duc (il devait devenir grand-duc à la fin de sa vie, en 1569). C'est pourquoi il chercha à y attirer des professeurs de premier plan, et parvint en effet à recruter des savants médecins comme Realdo Colombo, Gabriele Falloppio et Luca Ghini.

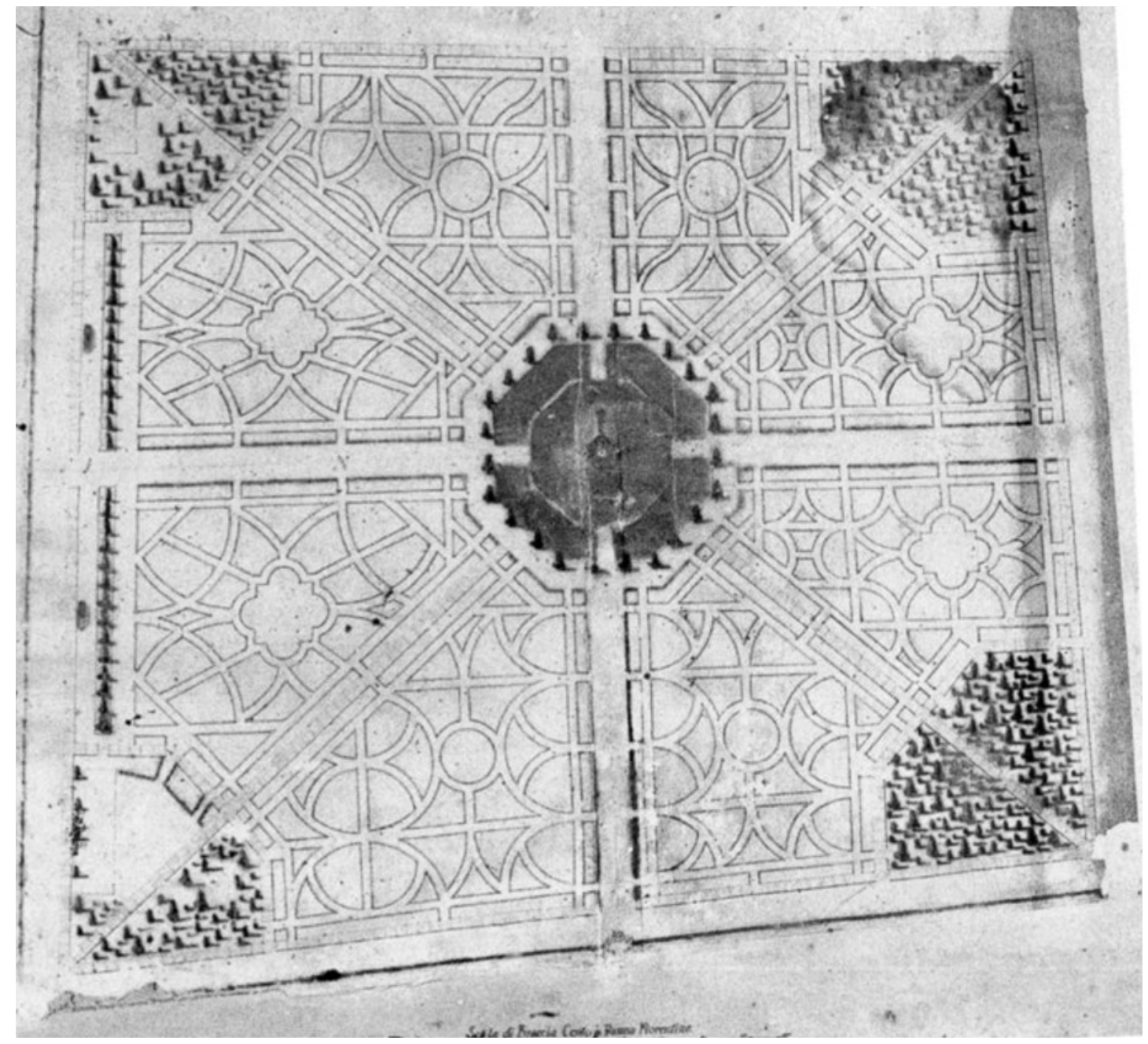

Fig. 1: Le jardin de San Marco tel qu'il fut conçu au $16^{6}$ siècle (d'après Luzzi/Fabbri 1993, 56). La géométrie en carré correspond à l'une des trois formes parfaites recommandées pour la réalisation de jardins par les théoriciens de l'architecture renaissante Alberti et Martini. Le dessin des allées et des massifs semble confirmer que la fonction esthétique et représentative du jardin primait encore largement sur son statut de lieu de recherche médicale et naturaliste. En suivant une interprétation néo-platonicienne, on peut supposer que la géométrie du jardin reflète la perfection du cosmos. A moins que les quatre côtés ne symbolisent les quatre humeurs de la tradition hippocratique, humeurs dont l'équilibre produit la santé. 
En 1544 donc, Luca Ghini, peu satisfait des conditions dans lesquelles il pouvait exercer la botanique pratique à Bologne, ${ }^{12}$ accepta l'offre du grandduc de Toscane de venir enseigner la «science des simples» à l'université de Pise, une offre assortie de la position rémunératrice de médecin du grandduc et de la possibilité de disposer d'un jardin botanique près de l'université. ${ }^{13}$ La création d'un tel jardin permettait de compléter, par une étude des plantes in vivo, un enseignement jusque-là essentiellement livresque, basé à Pise sur la lecture de Dioscoride dans une traduction latine publiée en 1544 par Pietro Andrea Mattioli et souvent rééditée depuis. ${ }^{14} \mathrm{~A}$ cet atout pédagogique s'ajoutait la possibilité de développer, au jardin, de nouveaux types de recherches sur les différentes phases de la vie des plantes, en suivant là encore un précepte de Dioscoride.

Luca Ghini, qui avait déjà commencé à former à Bologne son impressionnante phalange de disciples, vint à Pise accompagné de ses élèves Andrea Cesalpino, Bartolomeo Maranta et Luigi Anguillara, avec lequels il fit des herborisations et des collectes de spécimens minéralogiques et zoologiques. ${ }^{15}$ Il ne resta qu'une dizaine d'années à Pise,${ }^{16}$ mais cela suffit pour y imposer durablement un nouveau style d'enseignement, où la théorie était complétée par l'observation in vivo, ${ }^{17}$ et où l'étude des autorités, en l'occurrence de la Materia Medica de Dioscoride, n'était plus que le point de départ d'un indispensable travail de vérification, de correction et d'expansion du savoir sur les plantes. ${ }^{18}$ Son enseignement fut poursuivi par Andrea Cesalpino, titulaire de la chaire des simples de 1555 à 1569, puis de la chaire de médecine pratique jusqu'en $1592 .{ }^{19}$ C'est donc à Pise que Cesalpino élabora son œuvre botanique, naturaliste et pharmacologique, et qu'il publia son célèbre De plantis (1583), qui passe pour l'acte fondateur de la botanique systématique.

12 Il semble qu'il ait considéré que les jardins dont il pouvait disposer à Bologne, soit le jardin pharmaceutique et le jardin du couvent de San Salvatore, étaient trop petits ou malcommodes. Il avait d'ailleurs recours au jardin de ses beaux-parents Sarti.

13 Sur l'histoire du jardin de Pise, voir Garbari/Tongiorgi/Tosi 1991.

14 Bellorini 2016, 24.

15 Engelhardt 2011, 60. Parmi les autres élèves formés par Ghini à Bologne figuraient Pierandrea Mattioli et Ulisse Aldrovandi.

$16 \mathrm{Il}$ devait retourner à Bologne en 1555 pour y mourir un an plus tard. Parmi les botanistes formés par Ghini à Pise figurent le Flamand Matthias de Lobel (1538-1616) et le Français Pierre Pena (1535-1605).

17 Luzzi/Fabbri 1993, 49.

18 Bellorini 2016, 58-65. Ghini fut par ailleurs l'un des premiers à faire un usage systématique de l'herbier et des plantes séchées, usage qui fut repris par Aldrovandi, Cesalpini et par d'autres de ses élèves.

19 Il fut ensuite nommé médecin personnel du pape Clément VIII et professeur de médecine au collège de la Sapienza à Rome Voir les articles «Cesalpino» du Dictionary of Scientific Biography et du Dizionario Biografico degli Italiani. 
Cesalpino, qui était un aristotélicien, considérait la res herbaria d'un point de vue philosophique, et non plus médical, en cherchant les principes rationnels qui permettraient d'établir un système de classification basé sur l'essence même des plantes. Il fallait donc diviser les plantes en groupes en fonction de leurs similarités et dissimilarités, autrement dit de les ordonner selon leurs caractéristiques morphologiques. Le but était d'aider la mémoire du savant, confronté à un nombre croissant de plantes découvertes, en lui offrant des principes de classification stables qui ne soient pas fondés sur des accidents comme l'utilité, mais sur «l'âme végétative», c'est-à-dire sur un principe essentiel qui rende compte de la faculté de nutrition et de reproduction des plantes. ${ }^{20}$ La première étape de cette démarche fut la constitution, à partir de 1563 d'un herbier de 768 spécimens, qui permette d'avoir à sa disposition toutes les plantes en même temps, quels que soient leur temps de floraison ou leur localisation, et donc de faciliter le travail de comparaison. Les plantes ainsi sorties de leur contexte furent d'abord divisées en arbres et en herbes suivant leur mode de nutrition, puis subdivisées en fonction de leurs organes de reproduction (fleurs, fruits, graines). C'est ainsi que les 1500 espèces examinées dans le De Plantis furent réparties en 32 groupes.

Même s'il devait aussi faire une place pour les plantes du Nouveau Monde, Cesalpino ne cherchait au fond qu'à comprendre et à définir rationnellement des groupes que l'on percevait déjà intuitivement. Il se référait d'ailleurs à Théophraste, qui pour les espèces non-médicinales avait tenté une classification des plantes d'après leurs formes. Malgré tout, sa tentative ne fut guère appréciée de ses contemporains qui, à l'image de Caspar Bauhin, trouvèrent l'ouvrage certes savant, mais très obscur. Selon Cristina Bellorini, sa tentative pour émanciper la botanique de la médecine ne fut pas comprise par une communauté savante faite de médecins qui persistaient à étudier les plantes du point de vue de leurs vertus curatives. ${ }^{21}$ Sa façon de considérer que les propriétés médicinales des plantes relevaient de caractères accidentels, et non essentiels, a dû choquer. Brian Ogilvie a aussi fait remarquer que la plupart des naturalistes de la Renaissance ne voyaient aucune raison de pousser l'étude des plantes au-delà du sens commun, ou de l'experientia: à leurs yeux, Cesalpino imaginait des problèmes qui n'avaient tout simplement pas lieu d'être..$^{22}$ De manière générale, l'observation était peu considérée par les philosophes et par les savants, et c'est pourquoi la connaissance des simples fut longtemps abandonnée aux sages-femmes et aux apothicaires. Ce fait souligne le caractère innovant que constituaient les collectes de spécimens, la

20 Bellorini 2016, 71-84.

21 Bellorini 2016, 81.

22 Ogilvie 2006, 225-226

Gesnerus 74 (2017) 
pratique des herbiers, l'usage des jardins et les développements de l'illustration naturaliste.

La botanique médicale resta donc la pratique dominante et c'est à Padoue, où Luigi Anguillara (l'un des anciens élèves de Ghini) avait fondé un jardin des simples en 1545, que se regroupa la plus importante école de botanique médicale d'Italie avec Francesco Bonafede, Melchior Wieland, Pier Antonio Michiel, Giacomo Cortuso, Prospero Alpini et donc Anguillara. A partir de 1568, Bologne et Rome se dotèrent elles aussi de jardins pour l'étude des simples, de même que Naples (où enseigna Maranta, un autre élève de Ghini) et Ferrare, puis Messine ou encore Turin. ${ }^{23}$ Pise resta malgré tout un centre important pour l'étude des plantes. Fidèle à l'esprit de Cesalpino, Domenico Vigna, continua d'ailleurs à se référer dans les années 1610 et 1630 à Théophraste autant qu'à Dioscoride. Quant à Florence, la botanique médicale y fut pratiquée en premier lieu au Giardino dei Semplici de Santa Maria Nuova, ainsi qu'au Giardino botanico de San Marco. Les idées semées par Cesalpino ne devaient toutefois y germer que plus tard, au cours du XVIII siècle. C'est alors que son herbier fut en quelque sorte redécouvert, et successivement annoté par Micheli en 1717, puis par Giovanni TargioniTozzetti en 1737-38, et finalement par Ottaviano Targioni-Tozzetti en 1796.

\section{La botanique florentine entre médecine et art des jardins}

A partir du milieu du XVI ${ }^{\mathrm{e}}$ siècle, plusieurs facteurs stimulèrent le développement de l'étude des plantes à Florence. L'un, très particulier, était l'intérêt pour la botanique médicale des grands-ducs de Toscane, en particulier de Cosimo I ${ }^{\mathrm{er}}$ et de ses deux successeurs François $\mathrm{I}^{\mathrm{er}}$ et Ferdinand ${ }^{\mathrm{er}}$. L'autre, d'ordre plus général, fut le développement d'une nouvelle conception de l'art des jardins, qui en faisait des symboles de grandeur, de munificence et de raffinement.

Cosimo de Médicis aimait offrir à ses visiteurs de marque des médicaments qui étaient préparés, parfois par lui-même, dans la «fonderia» grand-ducale et qui étaient délivrés, avec des recettes, dans des boîtes à compartiments. ${ }^{24}$ En agissant ainsi, le grand-duc se présentait un peu à la manière d'un roi thaumaturge, soucieux de la santé de ses proches et de ses sujets. Un exemplaire de Dioscoride annoté par le duc Cosimo, témoigne de

23 Des chaires de démonstration des simples, ou si l'on veut d'histoire naturelle des médicaments, avaient été créées à Rome dès 1513 (mais fermée de 1527 à 1534 , suite au sac de la ville), à Padoue dès 1533 et à Bologne dès 1537.

24 Bellorini 2016, 20-23. 
sa connaissance des plantes médicinales. Une partie des expériences, et particulièrement des distillations auxquelles il se livra dans sa «fonderia», concernait aussi la fabrication de remèdes à partir de plantes, même s'il mena aussi des recherches à caractère alchimique. François I ${ }^{\mathrm{er}}$, fils aîné de Cosimo, établit à son tour une autre «fonderia», et même un Musée jouxtant le jardin botanique («Orto medicei») de San Marco, donc sur le site où Lorenzo de Medicis avait établi jadis son Académie des Arts. Ce laboratoire resta en usage sous le règne de son frère et successeur Ferdinand ${ }^{\text {er }}$, même si les expériences pratiquées relevaient désormais davantage de la médecine spagyrique de Paracelse que de la médecine herbale traditionnelle. ${ }^{25}$

L'autre facteur est lié à la politique de munificence inaugurée par Cosimo $\mathrm{I}^{\mathrm{er}}$ pour asseoir symboliquement son pouvoir sur Florence. Cette politique passait entre autres par la multiplication des villas et des jardins, jardins que l'on admirait surtout pour leurs statues, leurs grottes, leurs fontaines et leurs fascinants jets d'eau, mais aussi pour leurs étonnantes compositions végétales. Le jardin de Boboli, autour du palazzo Pitti, était le plus célèbre d'entre eux, mais des jardins du même genre existaient à la villa de Castello (mis en place dès 1537 par Niccolò Tribolo et Giorgio Vasari) et dans d'autres villas construites par Cosimo I ${ }^{\text {er }}$ et par ses successeurs. La villa de Castello comprenait ainsi un jardin des simples où une fontaine avec une statue d'Esculape, le dieu de la médecine, soulignait le fait que les Médicis étaient en charge de la santé de leurs sujets. Le reste du jardin était planté d'espèces rares en partie choisies par Cosimo lui-même. Au jardin de Boboli également existait un espace réservé aux simples, auquel Cosimo prenait un intérêt particulier. Une autre partie du jardin fut plantée d'espèces toscanes et exotiques suivant des choix souvent faits par le duc lui-même. ${ }^{26}$

François I ${ }^{\text {er }}$ et Ferdinand I ${ }^{\text {er }}$ héritèrent de la passion de leur père pour les plantes, comme en témoigne leur correspondance avec Aldrovandi. Dès 1569, François établit ainsi à Partolino un jardin qu'il peupla d'essences rares et qui fit l'admiration d'Aldrovandi. Ferdinand établit à son tour des jardins dans ses villas d'Artimino et d'Ambrogiana. Il envoya d'ailleurs en Crète Josef Goodenhuyse, dit Giuseppe Casabona, directeur de l'«orto botanico» de Pise depuis le départ de Cesalpino, à la recherche de plantes rares ou

25 Bellorini 2016, 33-39. Cet auteur estime que le fait que les grands-ducs de Toscane s'intéressaient de si près à la pharmacologie a contribué à rapprocher les apothicaires, qui exerçaient une profession manuelle et peu considérée, de la corporation, plus intellectuelle et beaucoup plus prestigieuse, des médecins. C'est pourquoi, l'édition de 1597 du Riccetario fiorentino, la pharmacopée florentine traditionnellement rédigée par des médecins, fut pour la première fois l'œuvre de deux médecins et de deux apothicaires nommés par Ferdinand Ier.

26 Bellorini 2016, 40-44. 
d'une utilité médicinale. Fondé en 1587, le jardin d'Ambrogiana fut le premier de Florence à présenter des fleurs, notamment des iris (symboles de Florence) et des tulipes, qui étaient une grande nouveauté à l'époque. Ce jardin s'inspirait de ceux décrits par les ambassadeurs européens en poste à Constantinople. ${ }^{27}$ Inutile de dire qu'à l'image des Médicis, bien des membres de la noblesse florentine créèrent leurs propres jardins, devenus des symboles de raffinement et d'élégance. Certains d'entre eux, comme Alamanno Salviati, Alessandro Acciaiutoli ou Niccolò Gaddi, devinrent même des connaisseurs. La diffusion de cet art des jardins entraîna un intérêt accru pour des plantes caractérisées par leur beauté et leur rareté, non par leur utilité. Autant qu'à leurs propriétés médicinales, on s'intéressait désormais à leur morphologie et à leur cycle de vie, de la germination à la fructification. Brian Ogilvie pense que la motivation esthétique fut d'ailleurs le principal facteur du développement de l'histoire naturelle à la Renaissance. ${ }^{28}$

\section{La botanique du XVII ${ }^{\mathrm{e}}$ siècle entre médecine et mécénat}

Même s'il demeurait en quelque sorte dans l'ombre portée de Pise, le Giardino Botanico de Florence connut d'abord une période de grande splendeur sous la direction de Giuseppe Casabona, qui l'enrichit de nombreuses plantes collectées dans toute l'Italie, en Sicile et jusqu'à Candie (Crète), mais aussi d'espèces des rives orientales et méridionales de la Méditerranée, voire importées du Nouveau Monde. L'étude des plantes méditerranéennes était dictée par les précédents de Théophraste, Pline et Dioscoride. Quant à l'intérêt pour les plantes du Nouveau Monde, il était régi par la curiosité et l'esthétique, deux facteurs orientant un nouveau regard sur les plantes, indépendamment de la médecine. Pour les Médicis réunir en un lieu toutes les plantes de la Création était aussi une façon de manifester leur puissance dans des jardins d'ailleurs remplis de symboles. Finalement, la pratique nouvelle des herborisations, introduite par Luca Ghini, assurait l'approvisionnement des jardins toscans en plantes indigènes, que l'on retrouvait désormais représentées jusque sur certains murs du Palazzo Vecchio.

Cette période faste, qui correspondait aux règnes des grands-ducs François I ${ }^{\text {er }}(1574-1587)$ et de Ferdinand I ${ }^{\text {er }}(1587-1609),{ }^{29}$ ne cessa pas avec la mort de ce dernier. Au début du XVII siècle, la cour florentine devint en

27 Bellorini 2016, 45-48.

28 Ogilvie 2006.

29 Corti/Maugini/Lippini 1986, 753. 
effet une pépinière de savants, notamment grâce à Galilée, devenu en 1610 mathématicien du grand-duc Cosimo II. Son successeur Ferdinand II, qui régna de 1621 à1670, est connu comme protecteur de l'Accademia del Cimento fondée en 1657 par son frère Leopold. C'était aussi un passionné de botanique et de météorologie, qui cherchait à appliquer les connaissances nouvelles au perfectionnement de l'agriculture. A une époque où se multipliaient les jardins privés remplis de plantes exotiques, il avait lui-même plusieurs botanistes à son service. L'un d'eux, le médecin Angelo Domini, dirigea l'Orto Botanico de 1654 à 1668 et rédigea un Plantarum Florentinos ortos ornatium (1662)..$^{30}$ Vers 1666, Ferdinand II créa la fonction, et le titre, de botaniste du grand-duc (Regio botanico) pour Paolo Boccone (1633-1704), naturaliste de Palerme qui fut l'un des premiers à se servir d'un microscope. ${ }^{31}$ Ce titre consacrait une discipline nouvelle que Cesalpino s'était déjà efforcé d'émanciper de la médecine. Il allait à un savant polyvalent, qui cultivait la médecine, la toxicologie, l'étude des volcans aussi bien que l'embryologie végétale ou la floristique, sans oublier l'histoire naturelle de la tarentule. ${ }^{32}$ Constatant que le Giardino Botanico, confié après 1668 à un simple jardinier, tendait à devenir un potager, Boccone préféra se doter de son propre jardin, «alla Badia di Castello» près de Florence. ${ }^{33} \mathrm{Il}$ s'y attaqua, là encore comme son prédécesseur Cesalpino, à une tâche de classification rendue plus nécessaire que jamais par la multiplication des espèces décrites. Ce travail aboutit à une étude intitulée Museo di piante rare della Sicilia, Malta, Corsica, Italia, Piemonte, e Germania, qui ne parut qu'en 1697, bien après son départ de Florence. ${ }^{34}$ En 1678, Boccone avait en effet accepté la position de lecteur et démonstrateur des simples que lui offrait l'université de Padoue. Quatre années plus tard, il devenait moine cistercien et se retirait du monde, peut-être pour pouvoir pratiquer l'histoire naturelle en toute quiétude.

Avec Cosimo III (1670-1723), souverain bien moins éclairé et plus bigot que ses prédécesseurs, le Giardino botanico de Florence perdit tout à fait son caractère scientifique et didactique pour se transformer en un simple établissement horticole et domestique, destiné à assurer la production de fleurs

30 Mattirolo 1899, 10.

31 Les autres botanistes étaient Benedetto Punta, directeur du jardin avant Domini, Francesco Casabona, fils de Giuseppe, Ferdinando et Filippo Domini, frères d'Angelo, et Pietro Maccetti, herboriste du grand-duc (Corti/Maugini/Lippini 1986, 755-757).

32 Typique de cet éclectisme sont ses Recherches et observations naturelles, dans lesquelles il soutenait entre autres la nature minérale du corail et des madrépores (1671) et son Museo di fisica e di esperienze (1697).

33 A cette époque, c'était en effet le jardin de l'hôpital Santa Maria Nuova, dirigé par Giuseppe Baldi, qui servait d'établissement scientifique.

34 Voir la notice d'Isabella Sermonti Spada dans le Dizionario Biographico degli Italiani (vol. 11, 1969). 
et de fruits pour la maison du grand-duc. De 1678 à 1718, il fut abandonné aux soins du jardinier Bartolomeo Vannini, puis de son fils Federigo. A Pise, Cosimo III soutint néanmoins les efforts de Michelangelo Tilli (1655-1740) pour agrandir le jardin botanique de l'université, dont il avait la charge depuis 1685. Il l'encouragea à y introduire des plantes asiatiques et africaines, fit venir des graines d'Amérique, et finança la construction de serres, parmi les premières du genre en Italie, qui rendaient possible la culture de l'ananas et du café ${ }^{35}$. Cette orientation pratique avait bien entendu ses avantages et ses inconvénients, à Pise comme à Florence. Pour faire sa cour au grand-duc, il était en effet de bon ton, voire nécessaire, de célébrer l'excellence et la richesse de ses jardins. De nombreux artistes se spécialisèrent ainsi dans la représentation des plantes. Ce fut le cas de Bartolomeo Bimbi (1648-1723), peintre naturaliste dont les représentations offrent aujourd'hui encore un témoignage précieux sur les végétaux présents à cette époque dans le grand-duché. ${ }^{36}$ Ces mêmes «agrumi, frutta, uve», inspireront les dessins de Tommaso Chellini (1672-1742), qui joueront d'ailleurs un rôle important pour les observations mycologiques de Micheli. ${ }^{37}$ Chellini avait en effet peint 339 figures colorées de champignons pour un volume où étaient indiqués le lieu de provenance, les modalités de végétation, parfois les propriétés, ainsi que les noms vulgaires des espèces. ${ }^{38}$ Cela le fit passer à son époque pour un «maestro di botanica», ${ }^{39}$ du moins dans cette partie très particulière qu'est la représentation des objets naturels, très à la mode à l'époque baroque.

Dans la seconde moitié du XVIII ${ }^{\mathrm{e}}$ siècle encore, les dessins des peintres florentins étaient appréciés loin à la ronde, sinon dans l'Europe entière. Un exemple en est donné par la demande du résident anglais à Venise, John Strange (1732-1799), lui-même éminent botaniste, qui réclama en 1771 des dessins naturalistes à Giovanni Targioni Tozzetti. ${ }^{40}$ Celui-ci chercha parmi les élèves d'Ignazio Hughford et de Giuseppe Piattoli quelqu'un qui soit susceptible de répondre à cette demande, et arrêta son choix sur Giuseppe Buonaiuti. Mais à cette époque, les grands-ducs de Toscane s'étaient déchargés depuis longtemps de la gestion quotidienne du Giardino Botanico, dont

35 Le catalogue des plantes du jardin de Pise fut publié par Tilli en 1723 (Catalogus Plantarum Horti Pisani).

36 Agrumi, frutte e uve nella Firenze di Bartolomeo Bimbi pittore mediceo, Firenze, Paretti, 1982.

37 Les dessins de Chellini sont conservés parmi les manuscrits de Micheli.

38 Libro di Funghi fatto da Tommaso Chellini cittadino fiorentino, v. Targioni Tozzetti, Notizie degli aggrandimenti delle scienze fisiche accaduti in Toscana nel corso di anni LX del secolo XVII, p. 28.

39 Targioni Tozzetti, Notizie..., p. 29.

40 Lettre de Giovanni Targioni Tozzetti à John Strange, Florence, 27 avril, 18 mai et 22 juin 1771 (British Library, Add. 23729). 
les clefs furent remises en 1718, à une Società botanica fiorentina formée peu de temps auparavant. D'autres acteurs avaient entretemps redonné vie à la botanique scientifique en Toscane. Et en premier lieu les religieux de l'ordre de Vallombrosa.

\section{La tradition botanique des moines de Vallombrosa}

En Toscane, une autre source traditionnelle d'intérêt pour les plantes résidait dans la passion monastique pour les plantes médicinales et pour la contemplation des œuvres du Créateur. A l'intérieur de l'ordre bénédictin de Vallombrosa, implanté dans la région de Florence depuis le $\mathrm{XI}^{\mathrm{e}}$ siècle, cette tradition remontait pratiquement à la fondation de l'ordre par Giovanni Gualberto, devenu par la suite le saint patron des forestiers. A l'époque de Galilée, qui étudia lui-même un temps au couvent, les moines de Vallombrosa s'intéressaient quelque peu à la géométrie et à la construction d'instruments. ${ }^{41}$ La botanique suivit bientôt avec Virgilio Falugi (1627-1707), auteur d'un guide de systématique intitulé Prosopopoejae botanicae (1697), ${ }^{42}$ et son disciple et collaborateur Bruno Tozzi (1656-1743). Ce fut l'origine d'une véritable tradition de botanique, et plus seulement de botanique médicale. A travers des figures comme Biagio Biagi (1670-1735), Salvatore Perier (+1771), Francesco Maratti (1723-1777) et Fulgenzio Vitman (1728-1806), cette tradition se maintint jusqu'au XVIII ${ }^{e}$ siècle et essaima dans des villes comme Rome, Pavie et Milan, au gré des différentes affectations de ces religieux. ${ }^{43}$ Les moines de Vallombrosa s'intéressaient aussi à l'agronomie, à l'image de Vitale Magazzini (+1606) dont le traité d'agriculture, publié pour la première fois en 1625, fut constamment réédité jusqu'en 1842.

Suivant un modèle établi par Paolo Boccone, les moines de Vallombrosa développèrent une démarche fondée sur l'exploration d'un territoire avec l'objectif de dresser des «cartes» botaniques qui ne prétendaient d'ailleurs pas forcément à l'exhaustivité. Il participèrent ainsi, à leur manière, à ce que Alix Cooper a appelé l'invention de l'histoire naturelle indigène. ${ }^{44}$ Entre 1700 et 1727 , Bruno Tozzi effectua par ailleurs une série de voyages en suivant

41 Diego de Franchi pratiqua les mathématiques et l'astrologie, de même que l'abbé Orazio Morandi, correspondant de Galilée. Leto Guidi construisit des lentilles, des lunettes et des télescopes. Son élève Mercuriale Prai enseigna la philosophie naturelle à Forli.

42 Prosopopoeiae botanicae: Pars prima sive nomenclator botanicus (1697); Pars seconda de plantis umbraelliferis proprie dictis, flore perfecto pentapetalo irregulari (1700).

43 Maratti devint en 1748 professeur de botanique pratique au Collegio Romano et directeur du jardin botanique de Rome. Vitman fut appelé à enseigner la botanique à Pavie avant de fonder, et de diriger, l'Orto botanico de Brera à Milan.

44 Cooper 2007.

Gesnerus 74 (2017) 
des itinéraires qui allaient de la Maremma à l'île d'Elbe en passant par les Apennins de la Toscane et se prolongeaient jusqu'en Vénétie, aux environs de Rome, ainsi que dans la campagne napolitaine. ${ }^{45} \mathrm{Au}$ cours de ses excursions, il établit des listes alphabétiques de plantes observées et cueillies avec des annotations sur leur localisation. Cette pratique, qui s'inspirait des principes décrits dans le Pinax de Bauhin, orienta la structure du «Catalogus plantarum etruriae et insularum adjacentium» et du «Tractatus de botanica», deux manuscrits richement illustrés qui serviront ultérieurement de modèle à Pier Antonio Micheli. L'œuvre, restée inédite, de Tozzi se caractérisait également par une attention particulière apportée aux champignons, aux lichens, aux algues et aux bryophytes ${ }^{46}$ une préférence qui sera aussi celle de Micheli. Comme Falugi et d'autres religieux de Vallombrosa, Tozzi se montra d'abord fidèle au système de Rivinus (Augustus Bachmann), qui avait entre autres caractéristiques celle de ne pas faire de distinction entre plantes arborées et herbacées. Il se convertit ensuite au système de Tournefort, peut-être sous l'influence de son élève Micheli.

Avant de décliner et d'évoluer vers l'agronomie et la sylviculture, la tradition botanique des moines de Vallombrosa a donc influencé la première génération de botanistes florentins du XVIIIe siècle, celle de Pier Antonio Micheli (1679-1737), dont Falugi et Tozzi furent les maîtres. Le jeune Micheli commença par confronter les plantes qu'il découvrait dans la région de Florence aux planches du Commentario a Dioscoride de Mattioli, qui faisait toujours autorité à l'Université de Pise, et que le libraire Felice Bonaiuti lui avait procuré dans une édition de 1585. Virgilio Falugi, dont la Propopopejae botanicae décrivait les plantes médicinales les plus simples, lui apprit ensuite à bien observer et à déterminer les espèces de plantes à l'aide de sa méthode, précisément conçue pour les débutants. ${ }^{47}$ Bruno Tozzi prit le relais et partagea avec Micheli sa passion de l'histoire naturelle, et particulièrement pour l'étude des plantes cryptogames, qui devint le socle d'une amitié durable. Bien qu'issu d'un milieu fort modeste (son père était teinturier), Micheli était désormais en mesure de jouer un rôle capital dans le développement de la botanique à Florence, et dans sa diffusion au sein de l'élite locale. Un moment clef dans ce processus fut la fondation, en 1716, de la Società Botanica Fiorentina.

45 La liste de ces voyages sera mise en ordre par Giovanni Targioni Tozzetti en 1771.

46 En témoignent un «Sylva fungorum depicta» daté de 1724 et un «Index Sylvae fungorum» non daté.

47 Targioni Tozzetti, G. (A.), Notizie della vita di Pier Antonio Micheli, Firenze, Le Monnier, 1858, p. 18-30. Corti/Maugini/Lippini 1986, 759-761. 


\section{De l'Orto di Boffi à la reprise du Giardino botanico (1706-1718)}

Au début du XVIII siècle, la pratique des herborisations commençait donc à se répandre à Florence. L'institutionnalisation de la botanique n'avait en revanche guère progressé depuis l'époque de Luca Ghini. L'un des premiers à s'en émouvoir fut le médecin Niccolò Gualtieri (1688-1744) ${ }^{48}$ conchyliologiste, botaniste et aussi peintre d'histoire naturelle à ses heures ${ }^{49}$. Alors qu'il était encore étudiant, il avait tenté, sans grand succès d'ailleurs, d'enrichir de plantes nouvelles le jardin de l'hôpital de Santa Maria Nuova, avec l'aide de Sebastiano Franchi, ${ }^{50}$ son futur directeur, d'Antonio Targioni ${ }^{51}$ et de Pier Antonio Micheli. Décidé à persévérer dans sa vocation, ce dernier bénéficiait de l'hospitalité de personnages comme le marquis Cosimo Da Castiglione (avec qui il herborisait parfois), les sénateurs Pandolfo Pandolfini et Filippo Buonarroti, le comte Lorenzo Magalotti et surtout le médecin Giuseppe del Papa (1648-1735). Ces notables avaient pris l'habitude d'inviter Micheli dans leurs villas de campagne afin de l'inciter à herboriser sur leur terres. Dal Papa, qui était médecin personnel de Cosimo III, parla de Micheli au grand-duc en des termes élogieux, ce qui lui valut d'obtenir en 1706 une pension annuelle de 80 écus, la charge d'aide-gardien au Giardino dei Semplici de Pise, avec l'obligation, plus morale que contractuelle d'ailleurs, de collecter des plantes pour ce jardin tout comme pour celui de Florence..$^{52}$

A la même époque, Gualtieri, Franchi, Micheli et l'abbé Gaetano Moniglia (1689-1749) ${ }^{53}$ décidèrent de s'associer afin de créer un petit jardin pour leur usage commun. Ils trouvèrent d'ailleurs un bout de terrain convenable Via Boffi, près de la Porta San Pier Gattolini, et ils se répartirent les charges de la manière suivante: Franchi - directeur; Moniglia - secrétaire; Gualtieri conservateur; Micheli - administrateur (provveditore). ${ }^{54}$

48 Médecin de la princesse Béatrice de Bavière, et brièvement de Gian Gastone de Medicis, éphémère professeur de médecine («morbis mulierum») à l'Université de Pise (1735-1738), Gualtieri possédait une belle collection de coquillages, dont il dédia le catalogue à FrançoisEtienne de Habsbourg-Lorraine. Il fut aussi l'auteur de Riflessioni sopra l'origine delle fontane (1725) Pour sa biographie, voir Morelli Timpanaro 1996.

49 Lami, Giovanni, Novelle letterarie, n. 10, Firenze, 6 marzo 1744, col. 149.

50 Originaire de Lucca, on ne sait pas grand-chose sur ce personnage, sinon qu'il a été médecin du grand duc Cosimo III. Voir Targioni Tozzetti, G. (A), Vita di PA Micheli, p. 31-33.

51 Oncle de Giovanni Targioni-Tozzetti.

52 Baccarini 1904 p. 50. Cet article, bien que marqué par son âge, est utile pour intégrer les informations données par Targioni père et fils dans leurs Notizie della vita di Pier Antonio Micheli, Firenze, Le Monnier, 1858.

53 Gaetano Moniglia était professeur «d'Istituzioni civili» et secrétaire de l'Accademia del disegno de Florence, cf. Antoine Jules Dumesnil, Histoire des plus célèbres amateurs français et de leurs relations avec les artistes. Tome I. Pierre Jean Mariette, 1694-1774, Paris, Jules Renouard, 1858, p. 54.

54 Novelle letterarie, n. 10, Firenze, 6 marzo 1744, col. 149. 
En 1710, les associés se disputèrent au sujet de l'avenir de leur jardin. Gualtieri et Moniglia étaient d'avis de rester indépendants; Franchi et Micheli souhaitaient au contraire un accroissement substantiel des moyens et du rayonnement de leur petite institution. ${ }^{55} \mathrm{Ils}$ entreprirent donc des démarches auprès de leurs connaissances les mieux en cour afin de fonder avec eux une Società botanica. Celle-ci vit le jour à l'automne de 1716 avec le concours de l'ecclésiastique Giuseppe Suares de la Concha (1683-1754), ${ }^{56}$ des sénateurs Cerchio de' Cerchi et Filippo Buonarroti, ainsi que des médecins Bartolommeo Gornia et Cipriano Targioni, tous membres de familles influentes. Don Bruno Tozzi, le grand botaniste de Vallombrosa, devait les rejoindre en 1719. Entretemps, en juillet 1711, Gualtieri avait démissionné de sa «charge» de conservateur du petit jardin de la Via Boffi, soit parce qu'il était en désaccord avec les projets de Franchi et Micheli, soit pour une autre raison, peutêtre liée à une opportunité de s'installer à Pise.$^{57}$ Franchi avait pour sa part obtenu la direction du jardin de Santa Maria Nuova (1712), où il invita aussitôt Micheli à venir planter des espèces indigènes dans le but d'en favoriser la propagation. Il suggéra aussi à Niccolò Gualtieri de lui fournir les doublets des espèces exotiques qu'il cultivait dans le jardin botanique de Pise. ${ }^{58}$ Gualtieri n'avait certainement pas rompu ses liens avec Florence, puisqu'il y loua en 1716 une salle pour des réunions d'amateurs d'histoire naturelle. Cette démarche, évidemment en lien avec la fondation de la Società botanica, montre qu'il avait probablement changé d'avis quant aux buts de cette association. ${ }^{59} \mathrm{Il}$ devait d'ailleurs succéder à Franchi à la direction du jardin de Santa Maria Nuova, avant que l'abbé Gaetano Moniglia ne le dirige à son tour jusqu'en 1730.

En 1718, la Società botanica reçut du grand duc Cosimo III la charge de gérer le Giardino botanico de San Marco fondé par son ancêtre Cosimo I ${ }^{\text {er }}$, et qui était désormais considéré comme dispendieux et peu utile. Micheli en assuma aussitôt la direction, qu'il convoitait depuis longtemps, ce qui provoqua peut-être la démission de Moniglia, qui eut dans tous les cas lieu en avril de cette même année. ${ }^{60}$ Ce jardin de San Marco était devenu depuis

55 Sur le changement d'attitude envers les sujets académiques, voir Cochrane 1961, spécialement ch. III-IV.

56 Noble florentin, il appartenait à l'ordre chevaleresque de Santo Stefano. Les origines de la famille sont portugaises. Un de ses ancêtres avait marié une soeur de Camilla Martelli, deuxième épouse du grand duc Cosimo III.

57 Baccarini 1904, 16, qui ne donne pas de référence précise pour cette information mais qui semble provenir d'une source sûre.

58 On ne sait rien des fonctions exactes de Gualtieri au jardin de Pise, où il travaillait peut-être comme volontaire. Voir Carranza 1974, 363.

59 Biblioteca biomedica, Carteggi [BBCF], Fondo Cocchi, 2.2.27, Carte varie riguardanti la Società botanica, c. (II) 1716.

60 Micheli, qui avait été nommé en 1706 gardien (custode) du jardin de Florence, chargé de son approvisionnement en plantes, se considéra dès lors comme surintendant de cette 
longtemps une sorte de jardin des délices, et Micheli eut fort à faire pour y changer les habitudes du jardinier Bartolomeo Vannini, puis de son fils Federigo. Les obstacles de toutes sortes que ceux-ci lui opposaient, et qui eurent tôt fait de l'exaspérer, sont mentionnées dans les Diari et dans divers documents concernant la Società botanica.$^{61}$ Le problème des rapports avec les jardiniers devait continuer à empoisonner la vie des botanistes qui lui succédèrent, tant celle de Targioni-Tozzetti avec le jardinier Spagnuolo (1731-1739) que celle de Saverio Manetti avec Paolo Boni (1739-1756) et surtout avec Uldericho Prucker (1756-1780).

A travers ces différentes péripéties, les quatre principaux fondateurs de la Società botanica avaient donc entrepris un renouvellement des institutions botaniques florentines, en particulier du Giardino botanico de San Marco et du Giardino dei Semplici de Santa Maria Nuova. Ce renouvellement marque le début d'un autre âge d'or de la botanique florentine, qui s'acheva avec la mort de Micheli (1737), survenue la même année que celle du grand-duc Gian Gastone, le dernier représentant de la lignée des Médicis.

\section{Le rayonnement de Pier Antonio Micheli (1718-1737)}

On a vu que la passion du jeune Micheli pour les plantes, mais aussi ses capacités, étaient devenues très tôt un objet de curiosité pour les notables florentins intéressés par la botanique. Plusieurs savants l'aidèrent aussi à surmonter les lacunes de sa formation littéraire, ou acceptèrent de corriger ses manuscrits avant publication. En échange de cette bienveillance, ces protecteurs se voyaient honorés par des dédicaces de plantes nouvellement décrites. Parmi ces soutiens figuraient plusieurs médecins comme Sebastiano Franchi et Giuseppe del Papa, médecins personnels de Cosimo III et de Gian Gastone de Medici, Cipriano Antonio Targioni (1672-1748), compagnon de nombreuses herborisations, ou encore Antonio Cocchi (1695-1758), qui devait se battre pour l'enrichissement du jardin botanique et pour l'élargissement du domaine d'action de la Società botanica. ${ }^{62}$

institution, office qui lui aurait été délégué par B. Parenti, alors qu'il y a tout lieu de croire que cette fonction n'était remplie que de facto, sur la base d'une délégation verbale ou tacite (Baccarini 1904, 231). La situation ne fut éclaircie qu'en 1718, lorsque la charge du jardin fut effectivement remise à la Società Botanica Fiorentina, qui en confia la direction (soprintende) à Micheli. Ce furent les seuls titres officiels jamais revêtus par cet important botaniste.

61 BIBF (Biblioteca dell' Istituta Biomedica di Firenze), Documenti, dissertazioni, lettere e bilanci della Società botanica fiorentina dall'anno 1724 all'anno 1774.

62 Corti/Maugini/Lippini 1986, 761-762. 
Grâce à l'émergence, à Florence, d'un milieu de botanistes, Micheli put donc cultiver cette science sans être passé par l'Université de Pise, ni même par le Studio de Florence, ce qui facilita sans doute son émancipation par rapport à la tradition de la botanique médicale. Ceci ne l'empêcha pas de développer tout un réseau de contacts et d'échanges, au départ avec l'appui de Bruno Tozzi, qui était lui-même en contacts avec Hermann Boerhaave, James Petiver, Hans Sloane et William Sherard (1659-1728). Celui-ci, qui était considéré comme le «prince des botanistes» de l'époque pré-linnéenne, accepta de rencontrer Micheli, alors âgé de 20 ans à peine, et se lia avec lui d'une amitié épistolaire durable. Les correspondances les plus fournies de Micheli sont néanmoins celles qui le lient à d'autres botanistes ou zoologistes italiens. ${ }^{63}$ L'un d'eux, Giovanni Girolamo Zanichelli (1662-1729), pharmacien à Venise, lui écrivit ainsi près de 150 lettres. Giuseppe Monti (16821760) professeur d'histoire naturelle et de pharmacologie à l'Institut des Sciences de Bologne, lui en adressa une centaine. Pour leur part, Michelangelo Tilli (1655-1740), professeur de botanique à l'Université de Pise, et Giuseppe Zinanni (1692-1753), naturaliste de Ravenne, lui en envoyèrent une cinquantaine chacun. Parmi les correspondants un peu moins réguliers figurent encore le maître Bruno Tozzi et le disciple Giovanni TargioniTozzetti, qui avaient pourtant avec lui des contacts plus directs, ainsi que le zoologiste et professeur de médecine Giovanni Bianchi (1693-1775) de Rimini.

Parmi les autres correspondants importants de Micheli figurent le «géologue» helvétique Karl Nikolaus Lang (1670-1741), le grand médecin néerlandais Hermann Boerhaave (1668-1738), ainsi que le médecin et naturaliste helvétique Johann Jakob Scheuchzer (1672-1733). Il échangea également quelques lettres avec les botanistes français Antoine de Jussieu et Sébastien Vaillant, avec le botaniste néerlandais Johannes Burmann et avec le savant polygraphe allemand Johann Heinrich von Heucher. Au niveau institutionnel, il entretenait des contacts avec quelques-uns des principaux jardins botaniques européens, en particulier ceux d'Oxford, Leyde, Paris et Vienne. Aucune trace en revanche d'une correspondance avec Tournefort, dont Micheli adopta de bonne heure le système de classification.

Authentique botaniste, Micheli était d'abord un systématicien convaincu de la nécessité d'une grande précision dans le choix des caractères per-

63 Un inventaire de la correspondance de Micheli a été établi pour nous par Daniela Parrini, d'après les lettres conservées dans le fonds Targioni-Tozzetti de la BNCF (Biblioteca Nazionale Centrale di Firenze). Ce fonds comprend 821 lettres reçues et 63 lettres écrites par Micheli. 
mettant la définition des espèces. Dans la première partie, seule publiée ${ }^{64}$, de son Nova plantarum genera (1729), il décrivit 1900 espèces végétales, dont 1400 nouvelles, pour la plupart des champignons, des lichens, des mousses et des hépatiques. Cet ouvrage était le fruit de voyages qui l'avaient mené à travers toute l'Italie (Vénétie, Emilie-Romagne, Latium, Abruzzes, Marches, Campanie, Pouilles et bien entendu Toscane) et même jusqu'en Prusse à travers le Tyrol, l'Autriche, la Bohême et la Thuringe (1708-09). Il fut d'autant plus apprécié que l'étude des cryptogames était encore peu développée à l'époque. ${ }^{65}$ On peut aussi penser que l'ignorance du latin, et donc de la littérature savante, était moins handicapante dans ce domaine que dans d'autres, puisque près de trois quarts des espèces décrites par Micheli étaient nouvelles.

A l'aide de deux microscopes assez primitifs, Micheli observa pour la première fois les organes sexuels (anthères et archégones) des mousses et les spores des champignons. ${ }^{66} \mathrm{~A}$ une époque où même un grand naturaliste comme Vallisneri pensait encore que les champignons étaient des excroissances morbides de certains végétaux provoqués par des lésions, Micheli se montra capable d'en décrire les premières phases de développement, à partir de leurs «graines». ${ }^{67} \mathrm{Il}$ fut le premier à décrire les asques et à voir les spores. Il put ainsi prouver que les champignons étaient capables de se reproduire et ne provenaient pas de la matière inanimée, comme beaucoup le soutenaient. Il détailla par ailleurs la structure des Graminées. Evidemment, ses descriptions restaient marquées par l'analogie de structure avec les plantes supérieures, de sorte que Micheli recherchait dans les champignons des organes similaires à ceux des fleurs. C'était aussi une conséquence de l'adoption du système de Tournefort, qui le poussait à classer les algues, et même des plantes dites lithophytes comme le corail, d'après la structure de la fleur. Linné, qui savait ce que signifiait le difficile examen des plantes inférieures, n'en témoignera pas moins de l'estime pour son collègue italien.

Le système de Tournefort fut aussi adopté comme référence pour la distribution systématique des espèces du Giardino botanico de San Marco. Il sera repris par les continuateurs de l'œuvre de Micheli, en particulier Giovanni Targioni-Tozzetti (1712-1783), son élève préféré, mais aussi par Giovanni

64 La seconde partie, consacrée aux algues, ne sera jamais achevée, même si Micheli parvint à réunir un matériau d'étude considérable sur le sujet.

65 En 1699 cependant, le peintre Tommaso Maria Chellini (1672-1742), lecteur de botanique au Studio Fiorentino, réalisa, on l'a dit, un manuscrit intitulé Nuovo Libretto di tutte le qualità dei Funghi miniati al naturale (BIBF, Mss R 210.16).

66 Sur l'œuvre scientifique de Micheli, voir les articles de Francesco Rodolico dans le Dictionary of Scientific Biography, d'Alessandro Ottaviani dans le Dizionario Biografico degli Italiani (vol. 74, 2010), ainsi que Schaechter 2000.

67 Corti/Maugini/Lippini 1986, p. 766-767. 
Lapi (1720-1788), responsable du Giardino dei Semplici de Santa Maria Nuova à partir de $1747,{ }^{68}$ et même, quoique de manière plus critique, par Saverio Manetti (1723-1784), successeur de Targioni au jardin de San Marco.

Dans un autre domaine, Micheli se distingua par des travaux de «physique végétale» sur l'appareil nutritionnel des plantes. Plusieurs de ses expériences, reprises plus tard par Targioni, lui semblèrent démontrer que le principe du développement des plantes repose sur l'attraction de particules homogènes, processus dans lequel l'eau joue un rôle essentiel. L'une de ces expériences consistait à placer des semences dans un mélange de verre pilé, de morceaux de coquille d'œuf et de poussière de charbon: arrosées régulièrement, les semences arrivaient à se développer. Cela démontrait, selon son élève Targioni, le pouvoir nutritionnel de l'eau. C'était la reprise d'une vieille idée de Van Helmont, qui semblait l'avoir accréditée par une expérience très connue consistant à mesurer l'augmentation de poids d'un saule pleureur régulièrement arrosé, mais planté dans une terre dont la quantité ne diminue pas. Targioni devait en déduire des recommandations adressées aux agriculteurs désireux d'améliorer leurs rendements. Selon lui, un labourage approprié devait permettre aux plantes de mieux profiter des particules nutritives, une recommandation qui se trouvait d'ailleurs en accord avec les idées de Duhamel Du Monceau. ${ }^{69}$

\section{La botanique entre médecine et histoire naturelle: Giovanni Targioni-Tozzetti}

Pour Micheli comme pour ses successeurs, la Società botanica fut tout à la fois un appui et une contrainte. Les cotisations de ses membres permettaient certes de couvrir les frais de fonctionnement du jardin, estimés à 550 écus (scudi) alors que la dotation annuelle du souverain n'était que de 350 écus. Mais d'un autre côté, le botaniste en titre partageait la gestion du jardin avec un bureau de quatre à six membres, parfois davantage, dont un président, un secrétaire, un ou deux provveditori, parfois un trésorier, sans parler d'un nombre variable de députés, conseillers ou censeurs..$^{70}$ Quoique théoriquement fixés à 20 en 1721 et à 50 en 1744 , les effectifs de la société étaient variables, de sorte que l'état de ses finances ne l'était pas moins. Or il fallait au minimum entretenir un jardinier et garantir la culture des plantes médicinales, ce qui n'était pas une mince affaire. Ainsi, un rapport de janvier 1730

68 Corti/Maugini/Lippini 1986, p. 768-769.

69 Giovanni Targioni Tozzetti, Ragionamenti sull'agricoltura toscana, Lucca, 1759.

70 La liste complète de ces «officiers» est donnée par Baccarini 1904, 252-254. 
constata que le nombre de simples cultivés avait décru de 1200 à 850 , et Micheli était invité à faire remonter ce nombre aux alentours des $1000 .{ }^{71}$ Le jardinier Federigo Vannini considérait en fait sa position comme une sinécure et son successeur Spagnuolo, nommé en 1731, préférait cultiver des agrumes et des poires dont il faisait commerce plutôt que des simples qui ne lui rapportaient rien.

La plupart du temps, la Società Botanica n'avait pas elle-même une activité débordante, même si elle ajouta la philosophie naturelle à son intitulé (1734) et prétendit même se transformer en Accademia fisico-botanica (1742). En tant que nouvelle société savante, elle s'occupait d'ailleurs autant, sinon davantage, de médecine, de sciences de la terre et de zoologie que de botanique..$^{72}$ Dans les années 1740 , la rareté des mémoires présentés semble indiquer qu'elle traversait alors une période creuse, consécutive à la disparition de ses fondateurs. La situation n'était apparemment pas meilleure du côté de l'hôpital de Santa Maria Nuova, où Antonio Cocchi fit un rapport dénonçant l'état d'abandon du jardin (1742). Il est vrai que cet établissement, dont la fonction était éminemment didactique, avait toujours été de dimensions très modestes. Sa situation ne s'améliora qu'à partir de 1747, avec la nomination comme directeur de Giovanni Lapi, par ailleurs lecteur de botanique au Studio Fiorentino..$^{73}$ Après avoir déploré le nombre infime de plantes dont il héritait (90 espèces de «vilissime erbe» selon lui) ${ }^{74}$ Lapi réorganisa en effet ce jardin selon la méthode de Tournefort, qui restera en place jusqu'à sa suppression en 1793. Le nombre de plantes avait entretemps crû à 900 en 1749, 1000 en 1753 et 1600 en 1765 , avant de diminuer à nouveau. ${ }^{75}$

A l'époque de Lapi, la gestion du Giardino de' Semplici de Santa Maria Nuova était en réalité chapeautée depuis Pise par Attilio Tilli, directeur du jardin botanique de l'université depuis 1740, en succession de son oncle Michelangelo Tilli. Il devait lui-même rester en place jusqu'à sa mort en 1781. Cette transmission dynastique eut pour effet de garantir une continuité de gestion, au prix d'une administration très personnelle de l'institution. Ce jardin hérita en 1746-1747, des collections d'histoire naturelle du médecin

71 Baccarini 1904, 233.

72 Dans les années 1734-1736, on y lut ainsi des communications relatives à la médecine (observations de Antonio Cocchi et de G. Civinini, enquêtes épidémiologiques de Giovanni Gentili), aux sciences de la Terre (géologie et fossiles du Valdarno) et à la zoologie (sur de nouvelles espèces de testacés) aussi bien qu'à la botanique proprement dite (Baccarini 1904, 247-248).

73 Lapi fut lecteur de botanique à l'école de pharmacie et de médecine de Florence, ou Studio Fiorentino, de 1747 à 1783. Ses Lezioni di Botanica nello Spedale di Santa Maria Nuova ont été publiées en 1776.

74 Baccini 1893.

75 Luzzi/Fabbri 1993, 53. 
florentin Niccolò Gualtieri, l'ancien co-fondateur de la Società botanica. Il fut bientôt équipé d'une petite station météorologique où Gian Lorenzo Tilli, le fils d'Attilio, était chargé de mesurer les précipitations et les températures.

A partir de 1737, la Toscane n'était plus administrée que par un Régent, car le nouveau grand-duc François II de Habsbourg-Lorraine, qui n'était autre que l'empereur François Irr , résidait à Vienne, où il demeura jusqu'à sa mort en 1765. Il n'en décida pas moins dès son avènement de réserver une partie du jardin de Boboli à l'établissement d'un nouvel Orto botanico (fig. 2), afin d'y cultiver les plantes nécessaires à assurer la continuité de l'enseignement de la botanique et de la pharmacologie (materia medica) du Studio fiorentino. ${ }^{76}$ On se souvient qu'à cette époque le jardin de l'hôpital Santa Maria Nuova était en crise et n'avait probablement même plus de directeur. Giovanni Targioni-Tozzetti, élève et successeur de Micheli qui enseigna la botanique médicale jusqu'en 1747 au moins, ${ }^{77}$ se trouvait maintenant disposer d'un Orto botanico dont les fonctions empiétaient largement sur celles du jardin de S. Maria Nuova, mais dont il pouvait faire à peu près ce qu'il voulait, étant seul maître à bord. Il en allait différemment au jardin de la Società botanica, dont la direction, qu'il exerçait en principe, était en fait collégiale. D'une certaine manière, la botanique médicale revenait en force, puisque Targioni était un médecin formé à l'Université de Pise, et donc un élève du médecin botaniste Michelangelo Tilli. Mais ce fut aussi le début d'un compagnonnage de la botanique avec l'histoire naturelle, qui était la science favorite de Targioni-Tozzetti. Cette science était justement en train de conquérir l'Europe, et elle commencera à s'institutionnaliser dans les années 1760, notamment en France, mais avec des connotations politiques qui n'existaient pas au même degré en Toscane. ${ }^{78}$ Par ailleurs, certains empires coloniaux, y compris la Russie, avaient entrepris depuis longtemps d'inventorier les ressources naturelles dont ils pouvaient disposer.

Dans une volonté de gouverner de façon éclairée, ou tout simplement afin de mieux connaître les ressources d'une Toscane qu'il administrait de loin, François II favorisa la multiplication des rapports en tous genres, y compris sur la flore et l'histoire naturelle du pays. Dans un tel contexte, les voyages d'observation ne pouvaient qu'être encouragés, et c'est sur mandat conjoint

76 Mattirolo 1899.

77 Corti/Maugini/Lippini 1986 parlent même (p. 769) de deux enseignements simultanés de la botanique médicale, confiés l'un à Targioni, l'autre à Lapi. Il semble toutefois que Lapi n'ait pas commencé à enseigner avant 1747. Quant à Targioni, s'il n'a pas cessé d'enseigner en 1747 , il n'est dans tous les cas pas allé au-delà de 1754 ou plus probablement de 1750-52.

78 Sur l'implication des naturalistes français dans l'amélioration physique et morale de la société, menée au nom de l'ordre naturel à travers d'innombrables initiatives littéraires, agronomiques, philanthropiques ou même à caractère politique, voir Spary 2000. 


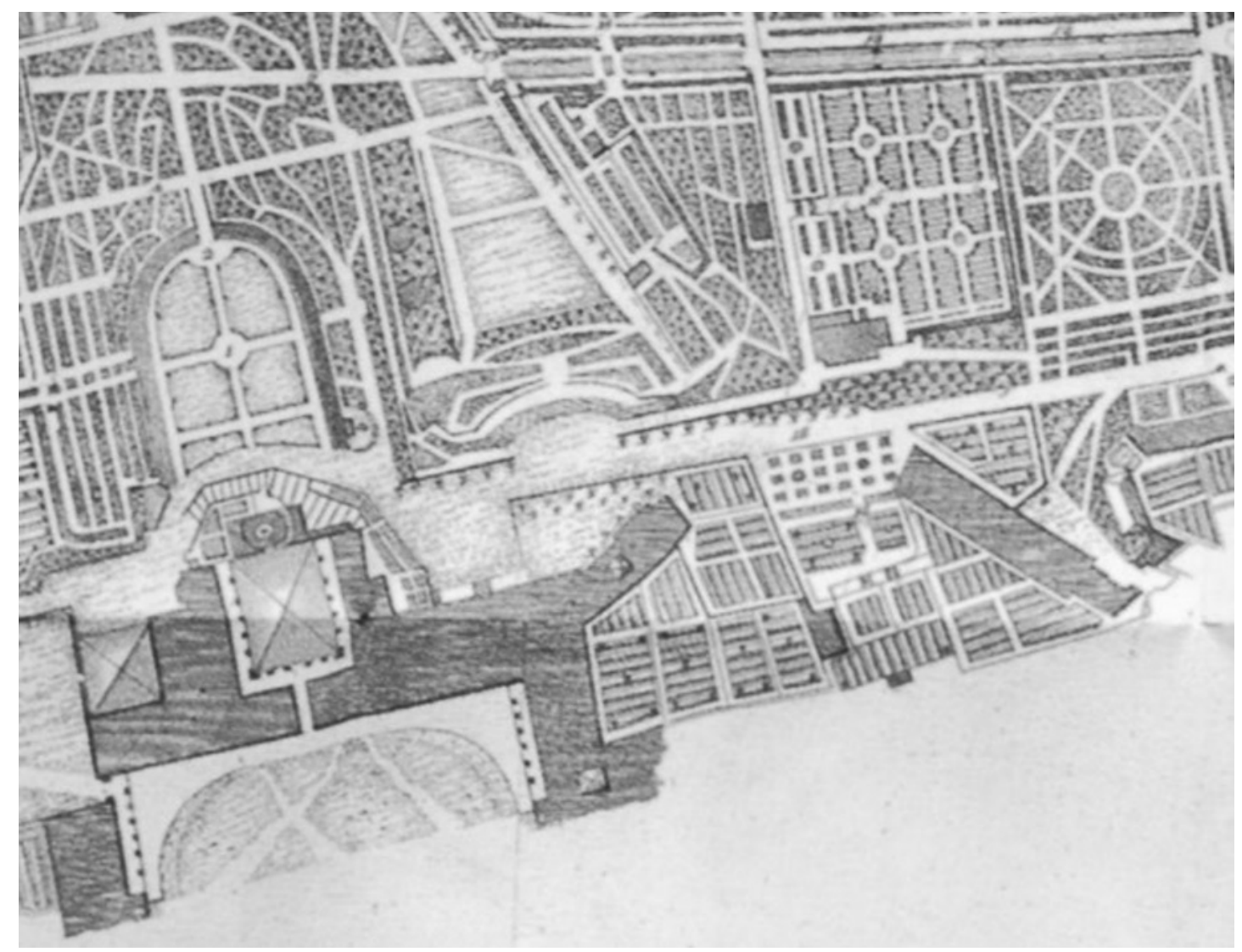

Fig. 2: Détail d'un plan du Jardin de Boboli (1789) montrant, en bas à droite, la portion affectée en 1737 à la botanique. Celle-ci se situait précisément entre le bâtiment principal (à gauche) et la partie traversée en oblique par un autre corps de bâtiment (à droite). Dès sa fondation au milieu du XVIe siècle, ce jardin avait eu des emplacements réservés aux plantes médicinales et aux plantes exotiques

de la Società Botanica et du Régent de Toscane, le comte de Richecourt, que Targioni-Tozzetti fit des excursions à Pise, Livourne, Volterra et Massa Marittima (1742), à Barga et Pietrasanta (1743) et à Monterotondo (1745). Les observations de ces voyages firent l'objet d'une première publication en trois volumes en 1751-1754, puis d'une seconde en 12 volumes en 1768-1779. Après une longue période d'intérêt pour les flores exotiques, les inventaires de plantes et de ressources naturelles indigènes revenaient donc en force. Les plantes récoltées au cours de ces herborisations ont naturellement enrichi les jardins de Florence, dont l'inventaire, esquissé par Micheli, finit par être publié en $1748 .^{79}$ Malgré tout, Targioni-Tozzetti abandonna en 1747 ses responsabilités à la tête de ces deux institutions, puis le secrétariat de la Società botanica afin de consacrer davantage de temps à la médecine, à ses travaux

79 Catalogus plantarum horti caesarei florentini. 
d'édition et d'inventaires ${ }^{80}$ mais aussi à ses recherches agronomiques et naturalistes, ainsi qu'à ses collections de plantes, de fossiles et de minéraux. Chargé en 1763 d'établir le catalogue des collections grand-ducales, ${ }^{81}$ il sera encore au nombre de ceux qui inciteront Léopold Ir (Pierre Léopold) à fonder en 1775 le Musée de Physique et d'Histoire naturelle (Imperiale e Reale Museo di Fisica e Storia Naturale), une institution aussitôt dotée de son propre jardin. Ce Musée, aussi surnommé «della Specola» parce qu'il était surmonté d'un observatoire astronomique et météorologique, devait à son tour organiser des expéditions naturalistes afin d'enrichir ses collections. Sa direction fut d'abord confiée au naturaliste et professeur de physique Felice Fontana (1730-1805).

La conclusion qui s'impose est que le développement, dès le début du XVIII siècle, de la botanique comme un loisir urbain a stimulé l'essor de nouvelles pratiques botaniques et naturalistes, en particulier la valorisation des espèces indigènes (floristiques et potagères, mais aussi minérales et zoologiques), ainsi que les expérimentations agricoles. Celles-ci sont venues s'ajouter à la curiosité et à l'ostentation aristocratiques, à l'enseignement des facultés de médecine, ainsi qu'aux recherches taxonomiques et physiologiques des botanistes spécialisés. Les jardins de Florence deviennent donc plus que jamais des institutions polyvalentes, où les attentes et les enjeux parfois contradictoires provoquent des tensions qui peuvent dégénérer en conflits larvés, voire ouverts. Le pouvoir grand-ducal, affaibli par le déclin des Médicis et par l'absentéisme de François II, peut bien encourager l'histoire naturelle et même créer un Musée qui lui soit consacré. Ce n'est plus qu'une composante d'un système institutionnel dont la complexité n'est pas sans évoquer celui d'une République de l'âge classique. ${ }^{82}$ Le manque de moyens, l'exiguiité des lieux de recherche et le petit nombre de naturalistes consacrés ne permettaient d'ailleurs pas toujours de répondre aux attentes de la société civile, ou du moins de son élite cultivée. De sorte que celle-ci a fini par prendre les choses en mains en créant l'Académie des Georgophiles.

80 Targioni-Tozzetti catalogua notamment la bibliothèque Magliabecchi de 1739 à 1777.

81 Catalogo delle Produzioni Naturali che si consevano nella Galleria Imperiale di Firenze.

82 Les sources reflètent parfois ces incertitudes. C'est ainsi que les contours de certaines fonctions, parfois âprement disputées, apparaissent ambigus ou mal délimités. Dans certains cas, il est même difficile de faire concorder les témoignages, notamment quant aux dates d'entrée en fonction ou aux attributions de certains lecteurs, professeurs ou directeurs d'établissement. On a déjà évoqué les désignations fluctuantes des jardins botaniques chez les contemporains, qui ont amené les historiens à se contredire plus d'une fois quant aux fonctions des uns et des autres. Le fait que certains botanistes aient dirigé successivement, ou même simultanément, deux établissements différents a d'ailleurs contribué à renforcer ces confusions. 


\section{Agriculture et expérimentation: l'Accademia dei Georgofili}

Au milieu du XVIII siècle, l'intérêt pour la botanique et l'histoire naturelle est donc bien implanté en Toscane, et pas seulement à Florence et à Pise. En 1754, Cortone se dote à son tour d'une Società Botanica modelée d'après celle de Florence. Les protagonistes principaux de cette société sont l'abbé Mattia Moneti et le religieux français J.-B. Royanel, qui réalisèrent des études sur la flore de Cortone. Moneti a laissé un herbier, que l'on peut considérer comme la contribution scientifique la plus remarquable de cette Société. Un autre botaniste issu de la Société de Cortone était Andrea Zucchini, qui devait diriger l'Orto agrario sperimentale de Florence dans les années 1790. A l'instigation du pharmacien Giovanni Domenico Stellanti, Pistoia se dota à son tour d'une Società botanica, qui entendait elle aussi mettre sur pied un jardin botanique..$^{83}$

A Florence même, on a vu que la Società botanica avait commencé dès 1734 à diversifier ses activités en direction de la médecine et l'histoire naturelle, mais aussi de l'agronomie. Le médecin Targioni-Tozzetti, qui en était la figure la plus emblématique, s'intéressait lui-même à tout ce qui était utile: médecine bien sûr, mais aussi agriculture, insalubrité de l'air, panification des céréales, lutte contre les famines, etc. ${ }^{84}$ Dans ses Ragionamenti sull'agricoltora toscana (1759), il décrivait les maladies des plantes importantes du point de vue agronomique, notamment celles qui affectent les céréales (ergot de seigle, carie, rouille, charbon). Il en élucida la nature parasitaire, due à des organismes végétaux microscopiques ${ }^{85}$ En 1767, il publia un Alimurgia ossia modo di rendere meno gravi le carestie, ouvrage de 367 pages consacré aux causes météorologiques des famines, qui fit l'objet d'une polémique avec Felice Fontana. ${ }^{86}$ De toute évidence, l'utilité s'imposait comme l'une des valeurs clés du siècle des Lumières, et l'une des justifications fondamentales de la pratique des sciences et des arts en Toscane comme ailleurs.

$\mathrm{Au}$ sein des élites, l'importance de l'agriculture dans l'économie locale, mais aussi la nécessité de remédier à ses carences, étaient de plus en plus clairement perçues. Cette évolution apparaît à la lecture des «Novelle Lette-

83 Maylender, M,. Storia delle Accademie in Italia, Cappelli, Bologna, 1929, vol. II, p. 278-279. 84 Sur l'œuvre scientifique de Giovanni Targioni-Tozzetti, voir l'article de Francesco Rodolico dans le Dictionary of Scientific Biography.

85 Corti/Maugini/Lippini 1986, 773.

86 Scarpellini 2006, 16-17. Parmi les autres publications de Targioni, il convient encore de citer: Prima raccolta di osservazioni mediche (1752); Prodromo della corografia e della topografia fisica della Toscana (1754); Ragionamento sopra le cause e sopra i remedi dell'insalubrità d'aria della Valdinievole (2 vol. 1761); Sitologia ovvero raccolta di osservazioni, di esperienze e ragionamenti supra la nature et qualità dei grani e delle farine per il panificio (2 vol., 1765). 
rarie», revue fondée en 1740 par Giovanni Lami et dirigée par lui jusqu'à sa mort en 1770. Dès sa première décennie d'existence, ce périodique consacra en effet de nombreux compte rendus à des ouvrages à caractère agronomique, essentiellement des classiques de l'agriculture, de l'horticulture et de l'histoire naturelle, ainsi qu'à des travaux relatifs à l'apiculture ou aux épizooties. ${ }^{87}$ Dans les années 1750 , cette tendance se renforce encore avec le traitement de questions agricoles, mais aussi de politique économique abordées dans une optique physiocratique ou néo-mercantiliste. C'est aussi l'époque où Saverio Manetti lance son Magazzino toscano (1754), où ces questions trouvent également une place ce choix.

La volonté de promouvoir le développement économique de la Toscane, et de contribuer à la réforme technique de son agriculture, suscita la création en 1753 de l'Accademia dei Georgofili, l'une des premières sociétés spécifiquement agraires d'Europe ${ }^{88}$ Principal instigateur de cette fondation, l'abbé Ubaldo Montelatici (1692-1770) s'efforça d'orienter le règlement de la nouvelle association avec l'aide de Targioni-Tozzetti, Saverio Manetti et Giovanni Lami. Son programme d'activités réformatrices s'inspirait directement d'un Ragionamento per far rifiorire l'agricoltura qu'il avait publié l'année précédente. Mais son point de vue n'était pas partagé par tous et Targioni y répondit en publiant ses propres Ragionamenti sull'agricoltura toscana.

Cet intérêt accru pour l'agronomie s'inscrit, dès les années 1740, dans un contexte de déclin de la théologie romaine qui aurait permis, selon certain historiens, au savoir médico-botanique de s'épanouir plus librement. ${ }^{89}$ C'est oublier que la pratique de la botanique, et plus encore de la médecine, était enracinée depuis longtemps dans la culture locale. De plus, l'abbé Montelatici s'inspirait lui-même de Ludovico Antonio Muratori (1672-1750), un religieux très érudit qui considérait qu'une meilleure éducation du clergé rural éclairerait non seulement la religion, mais aussi les paysans quant à la nécessité de moderniser leurs pratiques agricoles. ${ }^{90}$ Par ailleurs, les moines de Vallombrosa s'intéressaient depuis longtemps aux activités agricoles et arboricoles de leurs voisins, quitte à délaisser quelque peu leurs herbiers. L'un d'entre eux, Diamante Fuginelli (+1787), qui enseignait la philosophie

87 Pult Quaglia 2003, 562-564.

88 Le mouvement des sociétés d'utilité publique, ou des sociétés d'encouragement à caractère économique ou patriotique, avait débuté bien plus tôt, notamment en Angleterre, mais ces sociétés combinaient généralement le soutien aux arts (mécaniques) à celui de l'agriculture. Voir les études de cas regroupées par Stapelbroek/Marjanen 2012, ainsi que les ouvrages de Hudson 1972 sur l'Angleterre et de Bourde 1967 sur l'agronomie en France.

89 Pasta 1993, 486.

90 Pult Quaglia 2003, 566. 
et les mathématiques à Florence, fit ainsi des expériences de culture destinées à mettre en évidence les effets des différents types de terrains. Il fut d'ailleurs appelé par Charles Emmanuel III de Savoie à venir appliquer ses théories dans le royaume de Sardaigne. Son confrère Leopoldo Ducci (+1827) devait introduire en Toscane la culture de l'«andriolo», un blé trapu qui réussissait à mûrir dans le climat rigoureux du pays et pouvait ainsi se substituer avantageusement au seigle et à la «spelta» cultivés jusque-là. ${ }^{91}$ Luigi Fornaini (1755-1838) enfin, auteur d'un Della coltivazione degli abeti (1804), était un observateur méticuleux des domaines forestiers du couvent de Vallombrosa. Il devait d'ailleurs être chargé de les administrer pour le compte du gouvernement français lorsque celui-ci prit possession de la Toscane, et plus particulièrement des biens du couvent (1809). Il en tirera la matière d'un Saggio sopra l'utilità di ben governare e preservare le foreste (1825).

En réalité, c'est surtout l'appui manifesté à partir de 1765 par le nouveau grand-duc Pierre Léopold (Léopold Irr) qui devait faire de l'Académie des Georgophiles un instrument de réflexion pour une réforme de l'agriculture toscane. Il faut dire que ce soutien intervenait dans un contexte de famines répétées qui affectèrent le pays entre 1764 et 1767 . Un autre facteur déterminant, d'ordre général en Italie, fut la transformation, dans les années 1770, d'une culture savante qui demeurait encore essentiellement érudite, antiquisante et littéraire, en une culture beaucoup plus ouverte à l'observation et à l'expérimentation. Les observations de terrain, l'approche expérimentale et la recherche d'applications utiles firent à cette époque des progrès significatifs avec les travaux eudiométriques et chimiques de Felice Fontana, ${ }^{92}$ avec les collectes de terrain de Targioni-Tozzetti et finalement avec la création en 1775 du Reale Museo di Fisica comme lieu de recherches et de conservation équipé d'un observatoire, d'un cabinet d'instruments et d'un jardin botanique. Le langage de l'expérimentation se renforça au sein même de l'Accademia dei Georgofili, aboutissant à la transformation en 1783 du jardin de San Marco en Orto agrario sperimentale, ainsi qu'au lancement du Magazzino georgico. ${ }^{93}$

Ce développement de la culture expérimentale se faisait en quelque sorte au détriment de la simple observation naturaliste ${ }^{94}$, et au profit d'une appro-

91 En 1810, soit deux ans après la suppression des ordres religieux par les autorités françaises, Duci fut nommé administrateur des biens du couvent de Vallombrosa et réussit à maintenir ses vastes possessions en bon état de production.

92 En particulier ses Richerche fisiche sopra l'aria fissa (1775).

93 Magazzino georgico, cioè raccolta di notizie interessanti l'agricoltura e l'applicazione dei naturali prodotti alle arte utili, 1783-1785 (3 vol.).

94 Sur l'opposition relative entre expérimentation et observation naturaliste au XVIII ${ }^{\text {e }}$ siècle et plus largement entre experimentum et experientia dans la période «Early Modern», voir Spary 2000, 6 et 13-14, Ogilvie 2006, p. 17-24 et surtout Baroncini 1992. 
che plus physiologique et chimique des problèmes de l'agriculture. Parallèlement au lancement, dans les années 1785-1789, du Giornale fiorentino d'agricoltura (1785-89) d'Adamo Fabbroni et Jacopo Tartini, la chimie, connaissait entre 1775 et 1815 son premier essor dans toute la péninsule. ${ }^{95}$ Elle semblait capable de transformer l'agronomie et la physiologie végétale aussi bien que la médecine. Entre 1778 et 1790, la Toscane connut par ailleurs sa première réforme hospitalière, développée dans une optique hygiéniste et sanitaire.

A l'Académie des Georgophiles, les questions morales et politiques, encore en vogue dans les années 1770, firent donc place, vers la fin du siècle, à des concours plus orientés vers des questions pratiques. ${ }^{96}$ L'expertise technique des savants était de plus en plus recherchée, particulièrement dans les domaines de la chimie agraire et de l'œnologie.

\section{Entre Tournefort et Linné: la botanique de Saverio Manetti (1747-1782)}

Dans ce contexte de focalisation progressive sur l'histoire naturelle et sur l'agronomie, on peut se demander ce que devenait la botanique systématique florentine et comment elle se situait sur la scène internationale. L'examen structural du réseau de correspondance de Giovanni Targioni-Tozzetti offre un premier élément de réponse..$^{97}$ Outre son maître Micheli, on y trouve surtout des botanistes italiens qui lui sont très liés, tels que Giuseppe Monti (1682-1760) de Bologne et Michelangelo Tilli de Pise. Y figurent aussi les Florentins Niccolò Gualtieri, ci-devant directeur du Giardino dei Semplici, et Francesco Griselini (1717-1783), zoologiste, de même que Giovanni Bianchi (1693-1755) de Rimini. Egalement présents dans ce réseau le médecin franco-autrichien Johann Baptist von Bassand (1682-1740), le géologue John Strange (1731-1799), résident de Grande-Bretagne à Venise, le mathématicien Claudio Fromond (1703-1765), de Pise, et Ottaviano TargioniTozzetti (1755-1826), le propre fils de Giovanni. Parmi les savants de premier plan, on trouve surtout le géologue Ambrogio Soldani (1736-1808) de Sienne et le botaniste nîmois François Séguier (1703-1784), qui séjourna longtemps à Vérone ${ }^{98}$ Les deux caractéristiques fondamentales de ce réseau épistolaire

95 Cet essor est attesté par l'augmentation soudaine du nombre de spécialistes italiens affiliés aux principales académies des sciences européennes, ou alors signalés par le Dictionary of Scientific Biography et par le Historical Catalogue of Scientists de Robert M. Gascoigne (1984) (Source: recherche personnelle).

96 Pasta 1993, 498-499.

97 Pour l'inventaire de cette correspondance, voir Arrigoni 1986. Sur notre méthode de description statistique voir Sigrist 2008.

98 Séguier vécut de 1734 à 1755 auprès de son patron Scipione Maffei. 
sont donc la pluridisciplinarité et la prédominance nationale. Ces deux aspects reflètent l'intérêt de Targioni pour l'histoire naturelle et plus particulièrement pour les espèces indigènes.

Après 1747 , la charge de prefetto du Giardino botanico passa à Saverio Manetti (1723-1784), qui devait l'assumer jusqu'en 1780, conjointement avec celle de custodio de l'Orto botanico. Etant aussi de secrétaire de la Società botanica entre 1749 et 1758, Manetti devint ainsi, et pour plus de trente ans, la figure centrale de la botanique florentine. ${ }^{99} \mathrm{Il}$ avait été formé comme médecin à Pise, où il suivit les cours d'Antonio Cocchi, co-fondateur avec Micheli de la Società botanica de Florence. Sur le plan pratique, Manetti prit bien soin des jardins dont il avait la charge, et particulièrement de celui de San Marco, qui demeurait fondamentalement le même qu'à l'époque de Micheli, avec sa subdivision entre plantes médicinales d'un côté, plantes rares ou exotiques de l'autre, sans compter la partie réservée à la culture des fleurs et des agrumes (fig. 3). ${ }^{100}$ Pour maintenir son rang, Manetti entretint un bon réseau d'échanges avec les jardins de Londres (Philip Miller), Paris (Bernard de Jussieu), Göttingen (Haller), Leyden (Adriaan van Royen), Turin (Carlo Allioni) et Copenhague (Peder Ascanius). ${ }^{101}$ Ce réseau ne pouvait toutefois se comparer à celui établi jadis par Micheli.

En 1751, Manetti publia un Viridarium florentinum, catalogue des 1214 plantes du Giardino botanico dans lequel il donnait, comme ses prédécesseurs, sa préférence à la méthode de Tournefort. ${ }^{102}$ Comme il s'agissait d'un catalogue raisonné, il justifia sa préférence par la simplicité de cette méthode et par la nécessité de ne pas modifier inutilement les dénominations accréditées par les anciens et par la tradition médico-pharmaceutique. Toutefois, dans les cas où les dénominations de Tournefort ne concordaient pas avec celles d'auteurs plus anciens, Manetti considérait justifié d'introduire de nouvelles dénominations, en l'occurrence celles de Linné. Conformément au système de Tournefort complété par Sébastien Vaillant, il distinguait 22 classes de végétaux sur la base de la forme de la corolle et leurs subdivisions génériques et spécifiques selon la forme des fleurs et des fruits, sans négliger l'aide de la morphologie des feuilles, des branches, des racines ou de la forme générale de la plante. Cette méthode ne requérait donc point d'expert ni l'assistance d'un microscope pour pouvoir être appliquée. Elle

99 Le rôle de Manetti dans le développement de la botanique à Florence a été magistralement décrit par Stefani 2006, auquel ce paragraphe se réfère en grande partie.

100 Cellai \& al. 2012, 223-224.

101 Cellai \& al. 2012, 251.

102 Un catalogue des semences publié en 1747-1748 faisait déjà mention de 1200 espèces appartenant à 300 genres et 65 familles. Parmi elles, 325 plantes étaient spécifiquement destinées à l'enseignement (Cellai et al. 2012, 252 et 256). 


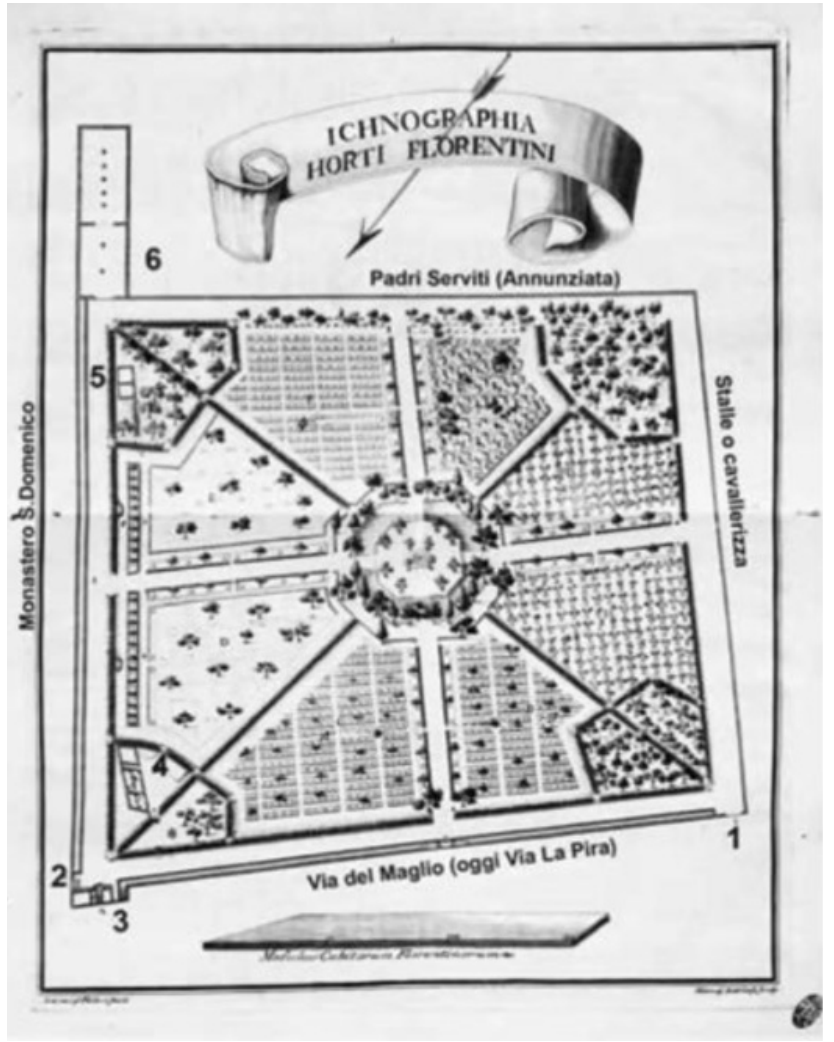

Fig. 3: Le Jardin botanicomédico-agronomique de San Marco à l'époque de Saverio Manetti. Les deux secteurs du haut regroupaient les plantes herbacées et les deux secteurs de droite les plantes arborées, toutes classées d'après Tournefort. Les deux secteurs de droite abritaient les plantes médicinales et les deux secteurs du bas des fleurs et des agrumes classés selon l'ordre alphabétique des désignations vernaculaires. Quant au numéro 4, il indique l'emplacement des serres chaudes et de l'angle du jardin consacré aux espèces exotiques, classées suivant le système de Linné (d'après Cellai \& al. 2012, fig. 4).

correspondait à une pratique pharmacologique de la botanique, où l'on a en effet besoin d'identifier les plantes selon une conformation qui soit en même temps un indice de leurs propriétés médicinales.

Selon Manetti, la méthode de Linné requérait au contraire un examen compliqué des pistils et du nombre d'étamines, une caractérisation exacte de la forme des stigmates, des anthères et des sacs à pollen, ce qui la réservait essentiellement aux études philosophiques. ${ }^{103}$ Manetti reconnaît certes l'importance fondamentale du système sexuel des plantes, mais cette considération ne doit pas justifier qu'on sacrifie la pratique botanique à la théorie. Pour lui, la stabilité d'une classification ne peut reposer que sur la forme et sur la structure de la fleur et du fruit. En résumé, le système linnéen, établi dans le Systema naturae (1735) et dans la Philosophia Botanica (1751), ${ }^{104}$ puis appliqué en 1753 dans le Species plantarum, comportait, selon Manetti, trois défauts:

1. Il est difficile à apprendre et à appliquer, car la détermination des plantes nécessite le recours au microscope, ce qui est peu pratique pour une activité médicale et pharmaceutique ordinaire.

103 Manetti, Viridarium, p. XII.

104 Stafleu 1971 considère que la Philosophia Botanica de 1751 offrait une présentation plus simple, plus claire et plus abordable du système de Linné. 
2. Il est artificiel et arbitraire, du fait de l'invisibilité des caractères essentiels choisis comme principe de classification. Il ignore d'ailleurs la division, fondamentale chez Tournefort, entre arbres et plantes.

3. Sa nouvelle nomenclature est susceptible d'introduire des confusions.

Pour toutes ces raisons, Manetti décida de maintenir la classification de Tournefort pour ordonner les plantes du Giardino Botanico, comme l'avaient déjà fait ses prédécesseurs Micheli et Targioni-Tozzetti, et comme le faisaient aussi Giovanni Lapi au jardin de Santa Maria Nuova et Attilio Tilli au jardin botanique de l'université de Pise.

Par la suite, les contacts épistolaires de Manetti avec Boissier de Sauvages, puis avec Linné lui-même, devaient peu à peu l'amener à réviser sa position. ${ }^{105}$ En 1756, il fit même paraître un Caroli Linnaei Regnum vegetabile iuxta Systema naturae... qui résumait les principales œuvres linnéennes, en particulier le Systema naturae, la Philosophia botanica et la $5^{\mathrm{e}}$ édition du Genera plantarum (1754). Si soudaine soit-elle en apparence, cette conversion n'était pas un cas unique. A Bologne, Giuseppe Monti (1682-1760) suivait exactement le même chemin que son collègue de Florence. Lui aussi avait commencé par préférer le système de Tournefort pour sa clarté et sa simplicité d'usage, accusant le système linnéen d'augmenter le chaos et la confusion par l'introduction d'une nomenclature nouvelle. Il avait également douté de l'efficacité ordonnatrice du système sexuel, relevant au passage plusieurs erreurs de classification commises par Linné. Mais ceci ne l'empêcha pas de changer d'avis dans les années $1750 .{ }^{106}$ Au niveau théorique donc, le système de Linné semblait devoir l'emporter assez facilement. Mais qu'en était-il au niveau pratique, compte tenu des contraintes de la gestion d'un jardin qui demeurait, pour partie, au service de la médecine et de la pharmacologie, et pour une autre partie au service de l'horticulture?

Les membres de la Società botanica restaient divisés. Deux documents de Targioni-Tozzetti, datant de 1758 et 1759, révèlent l'existence d'un débat que lui-même se gardait de trancher puisqu'il proposait de classer une partie du jardin suivant la méthode de Tournefort et une autre partie suivant la méthode de Linné, de sorte que les mêmes espèces puissent se retrouver dans

105 Stefani 2006, 281-283. Contrairement à une légende tenace, le contact épistolaire avec Linné fut très ponctuel: 3 lettres envoyée par Manetti, une seule reçue (voir The Linnaean Correspondence, an electronic edition prepared by Eva Nyström and the Swedish Linnaeus Society, Uppsala, and published by the Centre international d'étude du XVIII ${ }^{\mathrm{e}}$ siècle, Ferney-Voltaire).

106 Marta Cavazza, article «Monti, Giuseppe» in Dizionario Biographico Degli Italiani (t. 76, 2012). 
les deux parties du jardin !107 Il était même question, et cela fut confirmé par d'autres propositions faites à la même époque, de conserver le système pharmaceutique pour les plantes médicinales, et même de garder une classification alphabétique pour la partie du jardin consacrée aux agrumes et aux fleurs. ${ }^{108}$ Ce qui fut fait. Lors de ce débat, Manetti souligna les inconvénients des deux systèmes principaux, les plus graves étant encore, selon lui, ceux du système linnéen! A ses yeux, le choix d'un système théorique posait un problème d'exhaustivité s'il était suivi de façon systématique, car il risquait alors de souligner les manques de l'institution. ${ }^{109}$ En d'autres termes, le système de Linné était trop artificiel pour servir de guide à la connaissance des caractères naturels des plantes et particulièrement de leurs vertus médicinales. Il soulevait donc des difficultés d'ordre pratique. Du point de vue esthétique, il présentait de plus le grave inconvénient d'aboutir à placer parfois côte à côte des plantes d'aspects très différents. ${ }^{110}$ Le système de Tournefort permettait quant à lui de regrouper les plantes herbacées d'un côté, les plantes arborées de l'autre.

Sur un plan pratique, il paraissait donc difficile d'appliquer un système unique à une institution dont les fonctions demeuraient multiples. Certes, reconnaissait Manetti, la coexistence de deux systèmes de classification poserait de sérieux problèmes lors de la publication d'un catalogue. ${ }^{111}$ Il préconisait donc de «placer les plantes selon leurs affinités naturelles», ce qui signifiait en pratique regrouper entre elles les plantes annuelles, puis les pérennes, les marécageuses, les ombreuses, les alpines, les parasites et bien entendu les arbres. Selon sa formule, ce n'est pas aux plantes de nous enseigner le système, mais au système de nous permettre de mieux connaître les plantes. Le système doit d'ailleurs s'apprendre par les livres, et non pas au jardin, ${ }^{112}$ car l'ordonnancement de celui-ci répond avant tout à des considérations pratiques de gestion de l'espace.

A l'été 1763 , la Société botanique fut finalement à deux doigts d'imposer ${ }^{113}$ la classification linnéenne à un directeur plutôt réticent envers sa généralisation, alors même que son ralliement théorique à ce système ne faisait plus de doute. En fait, l'application de la méthode linnéenne fut limitée à une minuscule zone proche des serres, celle dévolue aux plantes rares et exotiques, le reste du jardin demeurant arrangé selon le système de Tournefort, du moins

107 BIBF: Diarj società botanica, f ${ }^{\circ}$ 249r; Stefani 2006, 285-286; Cellai \& al. 2012, 243.

108 Diarj, f ${ }^{\circ} 271 v-272 r ;$ Stefani 2006, 287.

109 Stefani 2006, 287-289; Cellai \& al. 2012, 243.

110 Diarj, f $349 \mathrm{r}-349 \mathrm{v}$.

111 Diarj, fo 351r-351v.

112 Diarj, f $\mathrm{f}^{\circ} 34 \mathrm{v}$.

113 Diarj, fo 307v, 5 agosto 1763; Cellai \& al. 2012, 244. 
pour sa partie systématique. ${ }^{114}$ D'où ce constat du minéralogiste suédois Johann Jakob Ferber, qui visita les lieux en 1771: «Le Giardino de' Semplici est grand, bien situé et arrangé d'après les deux systèmes principaux des plantes: celui de Tournefort, que suit M. Manetti, et celui de Linné». ${ }^{115}$

Saverio Manetti ne fut donc pas le grand rénovateur de la botanique florentine qu'on aurait pu imaginer, mais un savant médecin pragmatique, très conscient des enjeux pratiques de la gestion d'un jardin aux fonctions multiples. D'où le maintien, pour l'essentiel, du système de Tournefort, qui demeurait également en place au jardin de l'hôpital de Santa Maria Nuova. Giovanni Lapi (1720-1783) s'en servait encore en 1776 pour y enseigner la botanique aux élèves du Studio Fiorentino, comme il le faisait déjà trente années plus tôt. ${ }^{116}$

\section{Une botanique «républicaine»? Du triomphe de l'agronomie aux bouleversements de la période française (1783-1814)}

Le départ de Saverio Manetti fut l'occasion d'une réorganisation complète des institutions botaniques florentines, la principale étant la transformation du Giardino botanico en Orto agrario sperimentale. Dès 1773, le grand-duc Pierre Léopold avait proposé, au vu des difficultés financières rencontrées par la Società botanica, de la réunir avec l'Accademia dei Georgofili en une seule institution qui s'intitulerait Académie de botanique et d'agriculture. Pour rédiger un projet d'union, on nomma une commission comprenant des représentants des deux sociétés, mais le projet n'aboutit pas. La Società botanica finit d'ailleurs par se dissoudre entre 1780 et 1783, abandonnant la direction de son jardin à l'inusable Giovanni Lapi, qui y transféra les démonstrations de botanique médicale qu'il donnait dans le jardin de Santa Maria Nuova depuis plus de trente ans. ${ }^{117}$ L'Académie des Georgophiles, hérita en 1783 de l'emplacement et en confia l'administration à son secrétaire Jacopo Tartini, à charge pour lui de le transformer en un jardin à vocation agronomique: l'Orto agrario sperimentale (fig. 4). Selon d'autres sources, c'est l'agronome Marco Lastri (1731-1811) qui aurait été chargé de cette tâche. ${ }^{118}$

114 La partie médicinale échappa en effet à ce dilemme et une quatrième partie du jardin demeura classée selon l'ordre alphabétique.

115 J. J. Ferber, Lettres sur la minéralogie, p. 126, citées par Stefani 2006, 291.

116 D’après ses Lezioni di Botanica nello Spedale di Santa Maria Nuova (1776)

117 Di Fazio/Luzzi 2010, 102.

118 Ainsi, selon Di Fazio/Lutti 2010 (p. 102), l'Orto agrario sperimentale fut successivement dirigé, de 1783 à 1801, par Giovanni Lapi, Jacopo Tartini et Andrea Zucchini. Les dates 


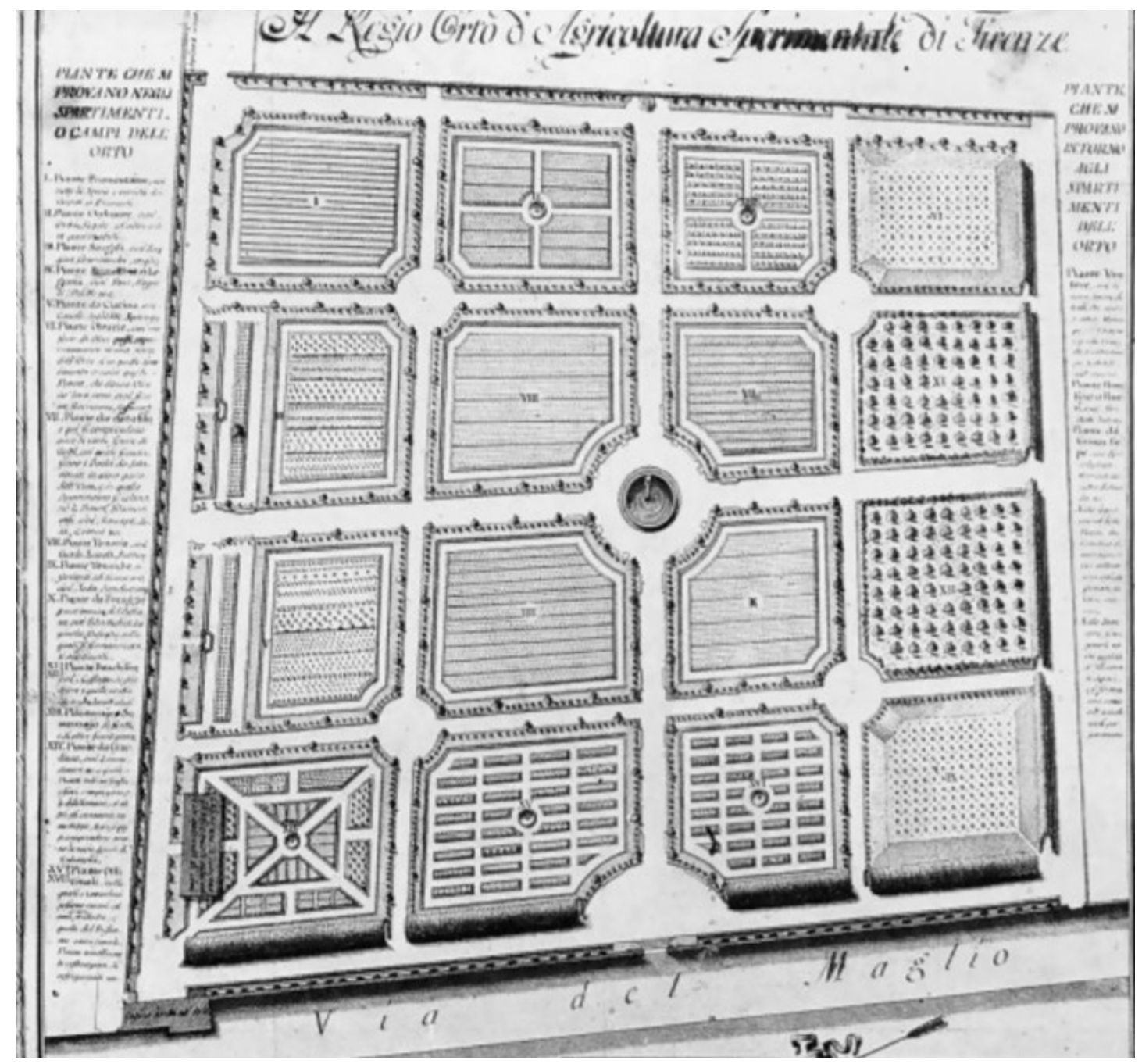

Fig. 4: L'Orto agrario sperimentale de l'Accademia dei Georgofili, ci-devant jardin botanique de San Marco après les transformations de 1783-1784 (d'après Di Fazio/ Luzzi 2010, fig. 3). La botanique systématique et la botanique médicale y étaient réduites à la portion congrue.

Une fois ce travail de transformation effectué, c'est l'abbé Andrea Zucchini qui fut nommé directeur de l'établissement, où il donnait également des cours d'agriculture.

Quoi qu'il en soit, l'agronomie triomphait. Son assise institutionnelle s'élargissait, car elle faisait consensus. Elle était associée en effet au réformisme grand-ducal ${ }^{119}$ tout en étant portée par les élites éclairées, et semblait

d'entrée en fonction ne sont pas précisées. Selon Nanni 2006 (p. 52-53), c'est Marco Lastri qui aurait succédé à Giovanni Lapi, transformant complètement le jardin avant d'être remplacé - en 1784 déjà - par Zucchini. La consultation d'autres ouvrages ne nous a pas permis de trancher cette question.

119 Le grand-duc Pierre Leopold entendait en effet conduire une politique économique et administrative réformatrice qui privilégiait le savoir-faire et la pratique sur la théorie ou la 
de surcroît correspondre au développement général des sciences expérimentales en Europe. De fait, le jardin des Georgofili abrita, dans ses 16 subdivisions, toutes sortes de plantes utiles: céréales et plantes panifiables; légumineuses et autres plantes de cuisine telles que choux, salades ou asperges; graines oléagineuses destinées aussi bien à l'illumination qu'à la savonnerie ou aux produits cosmétiques; plantes textiles (lin, coton) et tinctoriales, etc. Etaient également cultivées les plantes médicinales, les fourrages, les agrumes, les arbres fruitiers, les arbustes à baies, ainsi que les plantes à haies. ${ }^{120}$ Au tournant du siècle, l'agromanie semblait toucher toute l'Italie: un établissement semblable existait en effet à Padoue et d'autres allaient suivre à Turin (1798), à Bologne (1805) à Pavie (1806) et ailleurs encore.

Cette nouvelle orientation agronomique du jardin de San Marco rendit au Giardino dei Semplici de Santa Maria Nuova sa fonction première de lieu de démonstrations médicales. La direction en fut confiée à Ottaviano Targioni-Tozzetti (1755-1829), le fils de Giovanni qui venait d'être nommé lecteur de botanique médicale à la suite de son maître Giovanni Lapi. Comme son père et tant d'autres botanistes florentins, il avait étudié la médecine à Pise avant de s'intéresser à l'histoire naturelle et particulièrement à la botanique (médicale), qu'il apprit avec Giovanni Lapi et Andrea Zucchini à l'hôpital Santa Maria Nuova. ${ }^{121}$ Dix ans après cette nomination, il fut cependant décidé d'abandonner le Giardino de' Semplici (1793) et de transférer ses plantes au Jardin du Musée (Orto botanico), via Romana, qui prit à son tour l'appellation, si souvent utilisée par le passé, de Giardino dei Semplici. La plupart des 331 plantes transférées d'un établissement à l'autre étaient en effet des plantes médicinales, même s'il s'y trouvait aussi quelques arbres. ${ }^{122}$

De ce fait, le jardin du Musée devint, pour une partie au moins, un établissement d'enseignement médico-pharmaceutique, où Targioni poursuivit les démonstrations qu'il faisait jusque-là à Santa Maria Nuova. Mais l'établissement n'en demeurait pas moins sous la direction du médecin et naturaliste Attilio Zuccagni (1754-1807), qui avait succédé à Manetti en 1782 et qui, au début de son administration, avait considérablement augmenté le nombre de plantes cultivées, tout en les reclassant dans le système linnéen. ${ }^{123}$ Cette

philosophie. Son grand projet fut la mise en valeur des terres de la Maremma et du Valdichiana (Pisani/Nanni 1996).

120 Nanni 2006, 53.

121 Vergari 2006a, 21-25.

122 Fantoni/Cellai 2006, 148-149. Il fallut donc agrandir la surface disponible pour le jardin du Musée, travail qui ne fut achevé qu'en 1795.

123 De 1782 à 1784 , le nombre de plantes cultivées fut en effet porté de 1542 à 3734 espèces (Fantoni/Cellai 2006, 145). 
situation était elle-même un résultat indirect de la transformation du jardin de San Marco en lieu d'expérimentation agronomique. Le jardin du Musée ne correspondait plus en tout cas à l'établissement de recherche botanique dont avait rêvé le jeune Targioni. ${ }^{124}$ En attendant de parvenir un jour à ses fins, il préféra se concentrer sur la publication de ses Istituzioni botaniche (1794), dans lesquelles il décrivait toutes les plantes utilisées en médecine, en chimie, dans les arts et dans divers autres secteurs de l'économie. ${ }^{125}$ L'utilité restait donc à l'ordre du jour.

En fait, c'est l'invasion française du grand-duché qui allait redistribuer les cartes. Après une première conquête (1799), la Toscane fut enlevée en 1801 au grand-duc Ferdinand III pour être remise, sous le nom de royaume d'Etrurie, à la famille des Bourbon-Parme. Partisan de l'ancienne famille régnante, l'abbé Andrea Zucchini jugea alors préférable de s'éclipser définitivement, laissant la direction de l'Orto agrario (le jardin des Georgofiles) à son ancien élève Ottaviano Targioni-Tozzetti. Celui-ci reprit également la fonction de lecteur public d'agriculture, qu'il assumait en réalité depuis le premier départ de Zucchini deux ans plus tôt. ${ }^{126}$

En 1806, la création à Florence d'un Regio Liceo donna une nouvelle position à Targioni, qui devint titulaire de la chaire de botanique. Du même coup, il se retrouvait à la direction du jardin du Musée, aussi bien que de celui des Georgophiles, où sa position était désormais officielle. La botanique systématique, qui avait paru un temps menacée, reprenait maintenant tous ses droits, au moins dans le jardin du Musée. L'une des premières décisions de son nouveau directeur fut d'en reclasser les plantes selon le système de Jussieu et non plus celui de Linné. C'était une véritable révolution. En conséquence, la partie du jardin dédiée à la systématique fut subdivisé en 200 rectangles de 16 compartiments chaque (fig. 5). ${ }^{127}$

Ce changement d'orientation signale une influence française, dont le médiateur fut André Thouin, qui adressa pas moins de 24 lettres à son collègue Targioni entre 1789 et $1824 .^{128}$ Sur le plan politique par ailleurs, Targioni était vraisemblablement pro-français comme beaucoup de réformistes italiens des classes moyennes, qui avaient été privés de participation aux affaires sous les Habsbourg. Quoi qu'il en soit, l'empire français annexa

124 Vergari 2006a, 24. En conséquence de cette nouvelle fonction pédagogique, le nombre de plantes obtenues de semences passa de 221 en 1792 à 372 en 1794 et à 541 en 1797. Le total des plantes cultivées était de 4.366 en 1802 et de 6.000 en 1806 (Fantoni/Cellai 2006, 150-152).

125 Corti/Maugini/Lippini 1986, 775.

126 Zucchini étant malade, Targioni assumait en effet ces démonstrations d'agriculture depuis 1799.

127 Fantoni/Cellai 2006, 156-157.

128 Vergari 2002. 


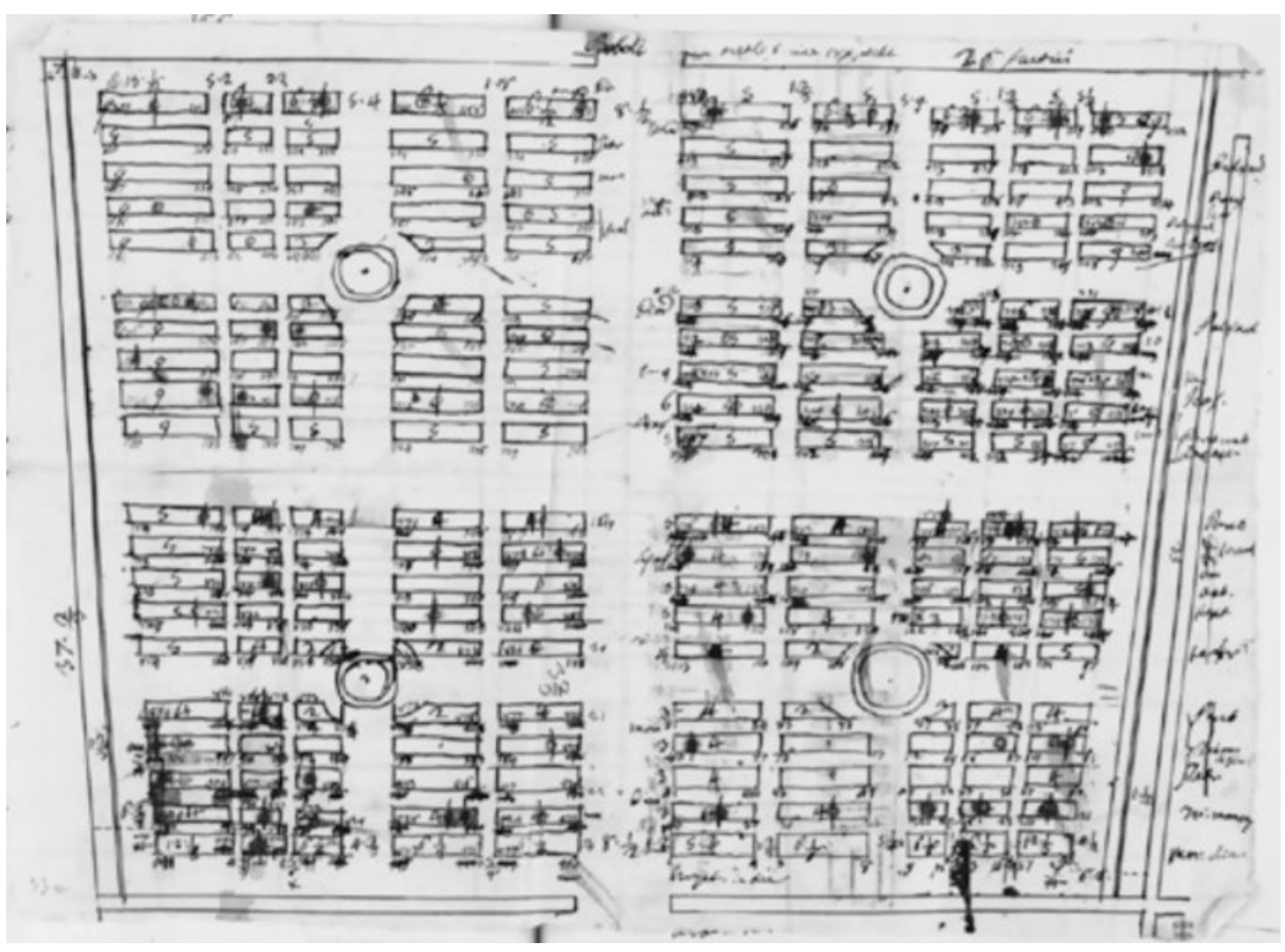

Fig. 5: La partie systématique du Jardin du Musée au début du XIXe siècle, d'après un croquis d'Ottaviano Targioni Tozzetti (D'après Fantoni \& al. 2006, 76). Elle ne comprend pas moins de 200 plates-bandes et 3200 compartiments.

le royaume d'Etrurie en 1807, Elisa Bonaparte recevant peu après le titre de grande-duchesse, avec la possibilité théorique de diriger un «Gouvernement général des Départements de la Toscane».

\section{Repli et redéploiement: la poursuite des traditions établies (1814-1839)}

La Restauration de 1814, qui ramena au pouvoir l'ancien grand-duc Ferdinand III, parut un temps menacer la position d'Ottaviano Targioni, dont la chaire de botanique au Musée fut supprimée. La direction du jardin du Musée lui fut également retirée et confiée au directeur de l'établissement Girolamo Bardi, un amateur de physique qui avait été attaché à la grandeduchesse de Toscane comme gouverneur des pages et chambellans. De ce fait, Targioni se trouva rejeté vers l'Orto agrario, donc il devait conserver la direction jusqu'à sa mort en 1829. Privé un temps de l'accès au jardin du Musée, il y fut à nouveau toléré à partir de 1817 et il y a même lieu de supposer qu'il ne tarda pas à en assumer à nouveau la direction scientifique 
de facto. Officiellement pourtant, sa juridiction ne s'étendait que sur l'Orto agrario des Georgophiles, ce qui semblait le condamner à se rabattre sur l'agronomie.

Conformément à la vocation première de ce jardin, Targioni favorisa les expériences portant sur des plantes à haute valeur agricole, en particulier les céréales (maïs, avoine, orge, épeautre, sarrasin, froment), dont il étudia les meilleures conditions de croissance de nombreuses espèces et variétés, ainsi que les possibilités d'acclimatation d'espèces peu ou pas cultivées en Toscane. ${ }^{129} \mathrm{Il}$ mena aussi des expériences de cultures de pommes de terre selon diverses méthodes, dans divers terrains, à diverses expositions et à l'aide d'engrais variés. ${ }^{130}$ Les cultures fourragères enfin firent l'objet d'expériences de rotation quadriennales des cultures avec l'introduction de prairies artificielles. ${ }^{131}$ Toutes ces recherches, bientôt surveillées par des instruments (thermomètre, pluviomètre, baromètre), se situaient dans le prolongement de son enseignement de l'agronomie, dont le contenu avait été publié dès 1802 sous la forme de Lezioni di agricoltura specialmente toscana.

En marge de la partie consacrée aux expériences agronomiques, Targioni s'efforça aussi de réaffecter une partie de l'ancien jardin de San Marco à la botanique systématique. ${ }^{132} \mathrm{Il}$ semble toutefois que les plantes de ce jardin soient restées classées selon la méthode linnéenne, qui avait déjà été adoptée après le départ de Manetti. ${ }^{133}$ C'était du moins le cas pour les plantes médicinales, qui continuaient donc à être cultivées dans ce jardin, comme dans celui du Musée, qui suppléaient tous deux à la disparition en 1793 du Giardino dei Semplici de Santa Maria Nuova. ${ }^{134}$ Quoi qu'il en soit, Targioni réussit à établir un catalogue des semences du jardin de San Marco avec l'aide de Gaetano Savi (1769-1844), un autre florentin qui était depuis 1810 professeur de botanique à l'université de Pise.

Sur le plan éditorial, l'inlassable activité botanique du second Targioni fut concrétisée en 1809 par la première édition de son Dizionario Botanico Italiano, où les appellations vernaculaires étaient mises en regard des désignations latines. Il est intéressant de relever en passant l'ambition déjà nationale de cette publication. Targioni publia par ailleurs un Catalogus vegetabilium marinorum (1826) qui résultait des travaux sur les algues de son père

129 Vergari 2006a, 26. Le nombre d'espèces examinées était de 25 entre 1801 et 1816 et de 72 en 1818 (NANNI 2006, p. 55-56).

130 Bigliazzi/Bigliazzi 2006, 56. Nanni 2006, 56.

131 Nanni 2006, 57.

132 Fantoni \& al. 2006, 79.

133 Cellai \& al. 2012, 318. En 1780, le jardin de San Marco conservait 370 espèces réunies en 207 genres.

134 Le nombre de plantes médicinales cultivées en 1805 était de 566, qui occupaient deux des seize subdivisions du jardin, soit quelque $2.000 \mathrm{~m}^{2}$ (Di Fazio/Luzzi 2010, $98-99$ et 103). 
Giuseppe et avant lui déjà de Micheli. ${ }^{135}$ Quant à son enseignement de la botanique médicale, qui paraît avoir eu beaucoup de succès à en juger par le nombre et la qualité de ses élèves, ${ }^{136}$ il fut lui aussi publié sous la forme de Lezioni di materia medica (1821). Targioni réunissait donc sur sa seule personne les différentes traditions locales d'étude des plantes, à savoir la botanique systématique, la botanique médicale et l'agronomie. Il était en revanche moins versé que son père dans les autres branches de l'histoire naturelle ou de la médecine, signe sans doute d'une relative spécialisation.

Une fois encore, l'examen du réseau épistolaire confirme ces orientations thématiques, tout en indiquant l'émergence d'une communauté nationale de botanistes. ${ }^{137}$ On y relève tout d'abord une relation absolument privilégiée plus de 200 lettres reçues - avec son concitoyen Gaetano Savi, successivement professeur de sciences au collège ducal de Florence, conservateur au Musée de Pise et professeur de physique, puis de botanique à l'Université de Pise. Savi fut l'un des grands systématiciens de sa génération, passant d'une Flora pisana (1798), à un Botanicon Etruscum (1808), puis à une Flora italiana (1818). ${ }^{138}$ Targioni reçut aussi près d'une centaine de lettres d'Antonio Bertoloni (1775-1869), professeur de botanique et directeur du jardin de Bologne, une cinquantaine de Domenico Nocca (1758-1841), qui occupait les mêmes fonctions à Pavie, et aussi de Filippo Re (1763-1817), professeur d'agriculture à Reggio Emilia et par ailleurs prédécesseur de Bertoloni à Bologne. D'autres botanistes italiens figurent parmi les correspondants réguliers de Targioni, en particulier : Giovanni Brignoli di Brunhoff (1774-1857), professeur à Modène; Antonio Campana (1751-1832), professeur à Ferrare; Giovanni Marsili (1727-1795), professeur à Padoue; Michele Tenore (17811861), professeur à Naples; Giuseppe Giuli (1764-1842), professeur à Sienne; et Luigi Colla (1766-1848), avocat à Turin. Certes, les individus cités appartenaient à trois générations différentes. C'est néanmoins tout un réseau de botanistes italiens qui paraît s'esquisser, une communauté nationale organisée autour d'un pôle toscan bicéphale incarné par Targioni à Florence et par Savi à Pise.

Sur le plan international, les échanges de Targioni sont plus fréquents avec André Thouin à Paris qu'avec Joseph Franz von Jacquin à Vienne, ce qui a sans doute une signification. On peut aussi noter ses contacts avec des natu-

135 Corti/Maugini/Lippini 1986, 777.

136 Parmi eux figuraient Gaetano Savi, Giuseppe Raddi, Cosimo Ridolfi, Giuseppe Giuli, Domenico Bruschi et Carlo Passerini, qui fut son assistant, ainsi que son fils Antonio Targioni-Tozzetti, qui l'assista à partir de 1821 et lui succéda en 1829.

137 Sur cet inventaire, voir Vergari 2002.

138 Il fut aussi l'auteur d'un Trattato degli alberi della Toscana (1801). 
ralistes comme Camillo Ranzani (1775-1841), directeur du Musée d'histoire naturelle de Bologne, Constantin Rafinesque (1783-1840), chercheur américain d'origine française, ou Giorgio Santi (1746-1822), directeur du musée d'histoire naturelle de Pise. A relever aussi ses relations avec des géologues comme Paolo Spadoni (1764-1826), professeur à Macerata, William Thompson (1761-1806), consul d'Angleterre à Naples, et Giovanni Arduino (17141795), professeur à Venise. Celles-ci montrent que les relations de la botanique avec les autres domaines de l'histoire naturelle n'ont pas cessé tout à coup. A Florence même, le type du naturaliste pluridisciplinaire restait représenté par le prêtre, médecin et bibliothécaire Giovacchino Carradori (1758-1818), qui pratiqua la chimie et la physique aussi bien que l'agronomie, la physiologie végétale et l'histoire naturelle en général.

D'autres figures de botanistes et naturalistes qui émergent sous la Restauration montrent la continuité des traditions de recherche établies à Florence au cours du XVIII siècle, mais aussi la lente émergence d'une communauté nationale italienne de chercheurs.

Une des figures de la continuité est Giuseppe Raddi (1770-1829), dont les origines sociales modestes rappellent celles de Micheli un siècle plus tôt. ${ }^{139}$ Et comme pour Micheli, il semble que ce soit encore la lecture d'un exemplaire du Commentario a Dioscoride de Mattioli qui a déclenché la passion du jeune Raddi pour la botanique systématique. Il partagea cette passion naissante avec Gaetano Savi, pas encore professeur de botanique à l'université de Pise, et il put surmonter les premières difficultés liées à la recherche grâce à la direction bienveillante d'Ottaviano Targioni-Tozzetti. En 1785, Raddi devint l'assistant d'Attilio Zuccagni, directeur du jardin botanique du Musée, ce qui lui permit de devenir l'un des conservateurs de ce Musée en 1795. Au cours de cette période, il effectua des excursions avec Savi et avec Targioni, accumulant un imposant matériel d'étude. En 1807, peu après la publication d'un premier mémoire sur les champignons, il fut cependant privé de son poste pour avoir refusé d'envoyer en France des spécimens qui lui étaient demandés. Rétabli dans ses fonctions à la Restauration (1814), il fut envoyé peu de temps après au Brésil afin d'y collecter des plantes destinées à enrichir les collections des Musées de Florence et de Pise. A son retour, il démissionna de ses fonctions afin de pouvoir se consacrer à la rédaction de son Plantarum Brasiliensium (1825). Il fut ensuite envoyé en Egypte pour y collecter de nouvelles espèces de plantes, mais rentra malade de son voyage et mourut peu après en 1829. Souvent à l'étranger, Giuseppe Raddi ne participa qu'assez modestement aux échanges entre botanistes

139 Sur Raddi, voir Corti \& al. 1987, p. 778-782, et Parrini (inédit). 
italiens du début du XIX ${ }^{\mathrm{e}}$ siècle. Dans son cas, ses principaux correspondants se nommaient Gaetano Savi (une fois encore), Giovanni Fabbroni (1752-1822), Vittorio Fossombroni (1745-1844) et Antonio Bertoloni (17751869), ainsi que Girolamo Bardi, le directeur du Musée de Florence. ${ }^{140}$

La carrière d'Antonio Targioni-Tozzetti (1785-1856), troisième du nom, s'inscrit également dans la continuité, à la fois dynastique et thématique. A la mort de son père Ottaviano (1829), il reprit en effet sa chaire de botanique médicale et la direction de l'Orto agrario. Formé comme médecin à l'université de Pise, comme l'avaient été son père et son grand-père, Antonio Targioni avait en effet débuté sa carrière comme adjoint à son père à l'hôpital de Santa Maria Nuova (1811-1824). ${ }^{141}$ En 1824, il était devenu lecteur de botanique dans ce même établissement. C'était une position d'attente idéale pour succéder à son père, dont il exerça les fonctions de 1829 jusqu'à sa propre mort en 1856. Représentant de la tradition médico-pharmaceutique, Antonio Targioni était aussi un scientifique de son temps, qui enseigna la chimie au Conservatoire des Arts et Métiers de Florence à partir de 1812. Il était donc capable d'employer ses compétences de chimiste à la recherche d'applications utiles à la pharmacie, à l'agriculture et à l'industrie. C'est dans le même esprit utilitariste qu'il pratiqua la botanique et l'agronomie, comme en témoigne la publication successive d'une Scelta di Pianti officinali pui necessarie a conoscersi (1824), d'une Raccolta di fiori, frutti ed agrumi più ricercati per l'adornato dei giardini (1825) et d'un Sommario di botanica e materia medico-farmaceutica per uso degli studenti di Farmacia (1828). Ses publications ultérieures concernent surtout des analyses d'eaux minérales, qui lui fourniront matière à pas moins de 29 opuscules.

Si Raddi illustre la botanique systématique et Antonio Targioni la botanique médico-pharmaceutique, la poursuite de la tradition agronomique toscane est assurée par Cosimo Ridolfi (1794-1865), grand propriétaire terrien à Meleto près de Florence. On a vu que tout en s'affirmant dès les années 1780 sur les plans institutionnels et éditoriaux, l'agronomie avait paru en mesure d'intégrer les dimensions théoriques et pratiques de la recherche sous l'égide des sciences expérimentales. C'était du moins l'espoir qu'avait exprimé Marco Lastri dans son monumental Corso di agricoltura pratica en 5 volumes (1787-90). ${ }^{142} \mathrm{Au}$ début du XIX ${ }^{\mathrm{e}}$ siècle, ce projet fut repris par Cosimo Ridolfi, lui-même devenu expert en chimie agricole. Elève d'Ottaviano Targioni (Targioni II), il chercha à faire de l'Accademia dei Georgofili,

140 Parrini (inédit)

141 Sur Antonio Targioni-Tozzetti, voir Vergari 2006b et Corti \& al. 1987, 782-784.

142 Pasta 1993, 499-500. 
où il était entré à 19 ans (1813), un instrument de développement de l'agriculture et de la viticulture dans les collines de Toscane. Il espérait ainsi promouvoir une agriculture originale, qui soit capable de répondre à la mécanisation et aux nouvelles techniques agraires utilisées dans les grandes plaines de l'Europe du Nord. En 1827, il fonda le Giornale agrario toscano avec Raffaelo Lambruschini et Giampietro Vieusseux, un organe destiné à perfectionner les conditions de l'exercice de l'agriculture et à améliorer l'instruction du peuple des campagnes. En 1834, Ridolfi créa un institut agronomique sur ses domaines de Meleto. Cet Istituto delle Cascine fut bientôt doté d'un professeur de chimie agricole ainsi que d'un champ d'expérimentation de vastes dimensions. A la même époque, l'espace confiné de l'Orto agrario de Florence apparaissait de moins en moins adapté à des expériences en grandeur nature qui requéraient bien davantage de place. Ce jardin fut finalement rendu en 1847 à l'hôpital de Santa Maria Nuova et retrouva aussitôt sa fonction première de Giardino de' Semplici. ${ }^{143}$

\section{Vers une botanique nationale (1839-1860)}

Le paradoxe politique de la Toscane fut de bénéficier, surtout après l'avènement en 1824 du grand-duc Leopold II, d'un des gouvernements les plus libéraux d'Italie, et l'un des plus favorables au développement d'une communauté scientifique nationale, sans pourtant parvenir rallier à sa cause la plupart des représentants de la classe moyenne, botanistes compris, qui se détournèrent finalement de la dynastie des Habsbourg-Lorraine.

Très caractéristique de cette évolution est le parcours d' Adolfo Targioni-Tozzetti (1823-1902), le propre neveu d'Antonio, que Cosimo Ridolfi engagea en 1857 comme professeur de chimie agricole dans son institut de Meleto. Comme les trois précédents représentants de la dynastie Targioni, Adolfo avait d'abord appris la médecine à l'université de Pise. Il a ensuite servi d'assistant à son oncle Antonio dans sa chaire de botanique et de materia medica de l'hôpital de Santa Maria Nuova, exactement comme Antonio avait été en son temps l'assistant de son père Ottaviano. ${ }^{144}$ En 1856, Adolfo Targioni fut nommé professeur d'histoire naturelle appliquée aux arts à l'Institut technique toscan. Le quatrième Targioni semblait donc bien parti pour continuer une tradition familiale de botanique et

143 La Société toscane d'horticulture se dota également en 1858 de son propre jardin expérimental.

144 Pour une biographie d'Adolfo Targioni-Tozzetti, voir Vergari 2006c et Corti \& al. 1986, 789-791. 
d'histoire naturelle vieille de plus d'un siècle. En 1859, il décida pourtant de rompre avec la dynastie régnante et s'en alla combattre les Autrichiens en Lombardie en espérant contribuer ainsi à l'unification de l'Italie. La même année, le grand-duc Léopold II abdiqua, ouvrant la voie à une réunion de la Toscane au nouveau royaume d'Italie. Projeté dans un contexte institutionnel nouveau, Adolfo Targioni décida alors, selon sa propre expression, de «déserter la botanique». Il renonça effectivement à son enseignement de la botanique médicale à Santa Maria Nuova pour se tourner vers la zoologie, particulièrement l'entomologie agricole, et vers l'anatomie comparée.

Sans forcément s'engager de façon aussi spectaculaire qu'Adolfo TargioniTozzetti, nombre de botanistes et de savants florentins des années 1840 et 1850 s'efforcèrent eux aussi d'apporter leur contribution à l'unification scientifique et politique de l'Italie. Dès 1839, ils jouèrent ainsi un rôle capital dans la fondation et dans les premiers développements de la Società Italiana per il Progresso delle Scienze. La première réunion, qui se tint à Pise en 1839, fut mise sur pied par trois savants qui bravèrent ce faisant l'hostilité du pape et celle des Autrichiens. L'un d'eux était Vincenzo Antinori (1792-1865), nommé en 1829 directeur du Musée de Florence, et qui avait rétabli les cours de physique et d'histoire naturelle supprimés après la période française. Le second était Giovan Battista Amici (1786-1868), qu'Antinori avait recruté comme professeur d'astronomie au Musée de Florence. Quant au troisième, c'était le fameux Paolo Savi, toujours professeur de botanique et directeur du Musée de zoologie de Pise. Après une seconde réunion à Turin, la Società Italiana tint à nouveau son assemblée générale à Florence en 1841, cette fois sous la présidence de Cosimo Ridolfi. On y décida notamment la mise sur pied, à Florence même, d'un herbier italien central, dont la charge fut confiée à celui-là même qui en avait suggéré la création: le jeune naturaliste palermitain Filippo Parlatore (1816-1877). ${ }^{145}$ En conséquence, celui-ci fut appelé l'année suivante à occuper la chaire de botanique et physiologie végétale du Musée de Florence, une chaire recréée pour l'occasion. Ce poste allait évidemment de pair avec la direction du jardin du Musée, où étaient conservés entre autres l'herbier de Cesalpino et de celui de Micheli. Parlatore ne tarda pas à faire de l'herbier italien central le vrai foyer botanique de la péninsule, qui rassemblait déjà 40000 espèces en 1846 et 78000 en 1848. En 1850, une collection de 80000 spécimens vint s'ajouter à cet herbier, lorsque le botaniste anglais Philip Barker Webb (1793-1854) décida de léguer son propre herbier au Musée de Florence.

145 Pour une biographie de Parlatore, voir Corti \& al. 1986, 791-797.

Gesnerus 74 (2017) 
Parlatore fut un directeur très énergique, qui devait faire du jardin de Florence un établissement important où étaient conservées pas moins de 11000 espèces vivantes, et qui fut par conséquent visité par de nombreux botanistes (fig. 6). ${ }^{146}$ Systématicien moderne, et représentant majeur de la géographie botanique, il réarrangea une partie du jardin selon la méthode de Candolle. ${ }^{147} \mathrm{Il}$ assuma par ailleurs un enseignement moderne de la botanique centré sur l'organographie et sur la physiologie des plantes. ${ }^{148}$ Il édita luimême quatre volumes d'une monumentale Flora italiana, une œuvre dont les six derniers volumes devaient être achevés par son assistant et successeur Teodoro Caruel (1830-1898). Il publia aussi des Considérations sur la méthode naturelle en botanique (1863), où il concluait qu'il n'existait aucun caractère absolument constant pour définir les genres, les familles, les classes et les divisions supérieures du monde végétal, mais seulement une structure complexe d'un petit nombre d'organes dont les caractères permettent d'établir des affinités et servent ainsi de base à l'élaboration d'une méthode naturelle. Il collabora enfin à la rédaction du Prodromus systematis naturalis regni vegetabilis de Candolle, dont il rédigea le volume 16 (1878).

Contrairement à ses prédécesseurs du XVIII siècle à Florence, Parlatore pratiquait une botanique systématique moderne, dégagée de la médecine, de l'histoire naturelle ou de l'agronomie. Grâce à un exceptionnel concours de circonstances, cette personnalité forte et habile obtint rapidement auprès du grand-duc Léopold II, qui était un amateur d'histoire naturelle, les appuis dont il avait besoin Il n'eut donc plus à s'inquiéter de trouver des soutiens dans la société locale, d'autant que le contexte politique et scientifique changea radicalement avec l'unité italienne. Parlatore put ainsi se concentrer sur l'inventaire des ressources végétales de l'Italie, ainsi que sur le développement de la science botanique en général. Il était devenu un spécialiste, un professionnel de la systématique. Les conditions dans lesquelles il œuvra avaient donc radicalement changé par rapport à celles de ses prédécesseurs du XVIII siècle.

146 Fantoni/Cellai 2006, 160.

147 Luzzi/Fabbri 1993, 64.

148 Le contenu de cet enseignement fut publié dès 1843 comme Lezioni di Botanica comparata. 


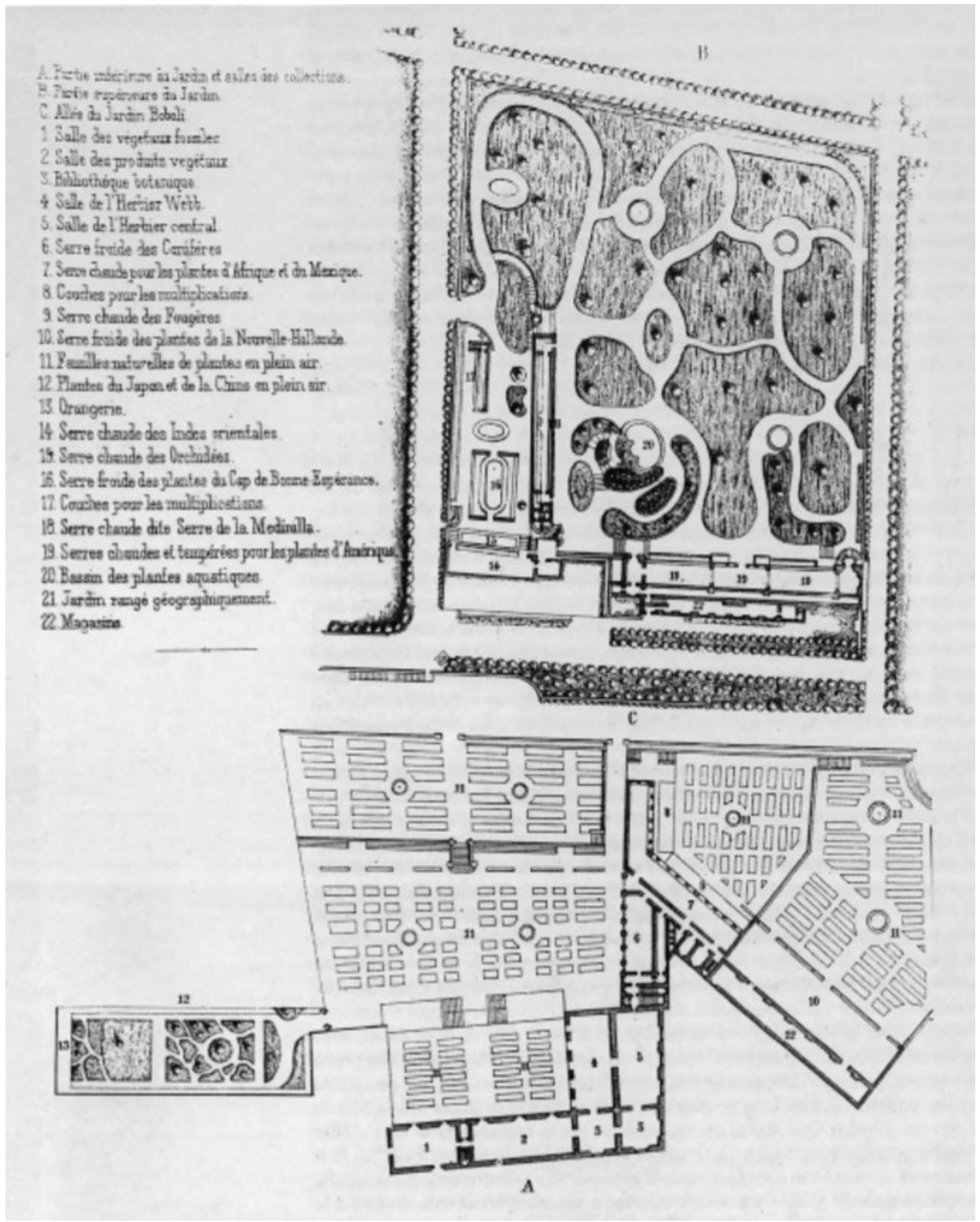

Fig. 6: Le jardin du Musée, tel qu'il se trouvait en 1874, à la fin du «règne» de Parlatore (d'après Luzzi/Fabbri 1993, 65). Il réunit toutes les caractéristiques d'un établissement moderne, prioritairement consacré à la systématique végétale. 


\section{Bibliographie}

Arrigoni, Tiziano, «Inventario del carteggio di Giovanni Targioni Tozzetti», Nuncius 1 (1986) 59-139

Baccarini, Pasquale, «Notizie intorno ad alcuni documenti della Società Botanica Fiorentina del 1716-1783 ed alle sue vicende», Annali di botanica 1 (1904) 225254

Baccini, G., «Uomoni illustri mugellani: notizie e ricordi, memoria presentata dell'abate Giovanni Lapi del Borgo S. Lorenzo al Granduca Pietro Leopoldo il dì 8 ottobre 1865», Bolletino storico letterario del Mugello 2 (1893) 13-16

Baroncini, Gabriele, Forme di Esperienza e Rivoluzione Scientifica (Florence 1992)

Bellorini, Cristina, The World of Plants in Renaissance Tuscany: Medicine and Botany (Farnham 2016)

Bigliazzi, Lucia/Luciana Bigliazzi, «I Targioni Tozzetti e l'Accademia dei Georgofili», in: Fausto Barbagli \& Daniele Vergari (éds), I Targioni Tozzetti fra '700 e '900 (Florence 2006) 47-66

Bourde, André, Agronomie et agronomes en France au XVIII siècle, 3 vols (Paris 1967)

Carranza, Niccolò, Monsignor Gaspare Cerati provveditore dell'Università di Pisa nel Settecento delle riforme (Pise 1974)

Cellai, Giovanna/Luciano Di Fazio/Luciana Fantoni/Paolo Luzzi, «Saverio Manetti e il "Giardino dei Semplici" di Firenze», Accademia Toscana, Scienze \& Lettere "La Colombaria”. Atti \& Memorie 76 (2012) 219-266

Cochrane, Eric, Tradition and Enlightenment in the Tuscan Academies (1690-1800) (Rome 1961)

Cooper, Alix, Inventing the Indigenous. Local Knowledge and Natural History in Early Modern Europe (Cambridge 2010)

Corti, Roberto/Elena Maugini/Patrizia Lippini, «Storia delle discipline botaniche dallo Studium generale all'attuale università», in: Luigi Lotti/Claudio Leonardi/ Cosimo Ceccuti (éds), Storia dell'Ateneo Fiorentino (Florence 1986) 743-797

Coturri, Enrico «La Scuola di Santa Maria Nuova di Firenze», Ospedali d'Italia. Chirurgia 11 (1964) 379-383

Di Fazio, Luciano/Paolo Luzzi, «La collezione dei Semplici nell'Orto agrario di Firenze del XIX Secolo», Museologia Scientifica n.s. 4 (2010) 98-104

Drayton, Richard H., Imperial Science and a Scientific Empire: Kew Gardens and the Uses of Nature, 1772-1903 (Ann Arbor 1993)

Engelhardt, Dietrich von, «Luca Ghini (1490-1556). Gründungsvater der neuzeitlichen Botanik im Kontext europäischer Wissenschaftsbeziehungen des 16. Jahrhunderts», in: Ingrid Kästner/Jürgen Kiefer (éds), Botanische Gärten und botanische Forschungsreisen (Aachen 2011) 57-80

Fantoni, Luciana/Giovanna Cellai, «Il giardino dell'imperiale e reale museo di fisica e storia naturale di Firenze dalle origine alla gestione di Ottaviano Targioni Tozzetti», Rivista di Storia dell'Agricoltora 46 (2006) 139-160

Fantoni, Luciana/Luisa Poggi/Giovanna Cellai Ciuffi/Paolo Luzzi, «I Targioni Tozzetti e il Museo di Storia Naturale», in: Fausto Barbagli/Daniele Vergari (éds), I Targioni Tozzetti fra '700 e '900 (Florence 2006) 67-80 
Garbari, Fabio/Lucia Tongiorgi/Alessandro Tosi, Giardino dei semplici - L'orto botanico di Pisa dal XVI al XX secolo (Pise 1991)

Hudson, Kenneth, Patriotism with Profit. British Agricultural Societies in the Eighteenth and Nineteenth Centuries (London 1972)

Lucarella, Agostino, Storia dell'Arcispedale di S. Maria Nuova di Firenze (Bari 1986).

Luzzi, Paolo/Fernando Fabbri, «I tre orti botanici di Firenze», in: Sara Ferri/ Francesca Vannozzi (éds) I giardini dei semplici e gli orti botanici della Toscana (Pérouse 1993) 49-68

Mannelli-Poggioli, Maria (éd.), Antonio Cocchi, Relazione dello Spedale di Santa Maria Nuova di Firenze (Florence 2000)

Mattirolo, Oreste, Cenni cronologici sugli orti botanici di Firenze (Florence 1899)

Morelli Timpanaro, Maria Augusta, Per una storia di Andrea Bonducci (Firenze, 1715-1766). Lo stampatore, gli amici le loro esperienze culturali e massoniche (Rome 1996)

Nanni, Paolo, «I Targioni Tozzetti e l'orto agrario sperimentale dei Georgofili», Rivista di Storia dell'Agricultura 46 (2006) 47-64

Ogilvie, Brian, The Science of Describing: Natural History in Renaissance Europe (Chicago 2006)

Palagi, Piero, «La Scuola Medica di S.Maria Nuova, in Firenze. Memorie-Testimonianze Prospettive», in: VII centenario della fondazione dell'Ospedale, Atti delle giornate celebrative (Florence 1988) 213-215

Parrini, Daniela, Le attività di un dimenticato "Ornamento d'Italia». Giuseppe Raddi, il naturalista, il conservatore, il viaggiatore (Pise; inédit)

Pasta, Renato, «L'Accademia dei Georgofili e la riforma dell'agricultura», Rivista Storica Italiana 105 (1993) 484-501

Pisani, Piero Luigi/Paolo Nanni, «Gli Orti agrari di Firenze», Rivista di Storia dell'Agricoltura 36 (2006) 69-93

Pult Quaglia, Anna Maria, «Le "Novelle Letterarie"et la cultura agronomica da Giovanni Lami a Marco Lastri», in: Carlo Ossola/Marcello Verga/Maria Antoinette Visceglia (éds), Religione, cultura e politica nell'Europa dell'età moderna. Studi offerte a Mario Rosa dagli amici (Florence 2003) 559-569

Scarpellini, Alba, «Giovanni Targioni Tozzetti. Uno scienziato al servizio della Toscana», in: Fausto Barbagli/Daniele Vergari (éds), I Targioni Tozzetti fra '700 e '900 (Florence 2006) 11-19

Schaechter, Moselio, «Pier Antonio Micheli, The father of modern mycology: A paean», McIlvainea 14 (2000)

Sigrist, René, «Correspondances scientifiques du XVIII ${ }^{e}$ siècle : présentation d'une méthode de comparaison», Revue Suisse d'Histoire 58 (2008) 147-177

Spary, Emma, Utopia's Garden. French Natural History from Old Regime to Revolution (Chicago 2000)

Stafleu, Frans A., Linnaeus and the Linnaeans: The Spreading of their Ideas in Systematic Botany (Utrecht 1971)

Stapelbroek, Koen/Jani Marjanen (éds), The Rise of Economic Societies in Eighteenth Century: Patriotic Reform in Europe and North America (Londres 2012) 
Stefani, Marta, «Linneo a Firenze. Saverio Manetti e l'ingresso del linneismo alla Società Botanica fiorentina», in: Ferdinando Abbri/Massimo Bucciantini (éds), Toscana e Europa. Nuova scienza e filosofia tra '600 e '700 (Milan 2006), 273-291

Vergari, Daniele, «La corrispondenza di Ottaviano Targioni Tozzetti», Nuncius 17 (2002) 91-163

Vergari, Daniele, «Ottaviano Targioni Tozzetti. Tra botanica e insegnamento: il maestro di une generazione di scienzati», in: Fausto Barbagli/Daniele Vergari (éds), I Targioni Tozzetti fra '700 e '900 (Florence 2006a) 21-29

Vergari, Daniele, «Antonio Targioni Tozzetti. Medico e scienzato nella Toscana dell'Ottocento», in: Fausto Barbagli/Daniele Vergari (éds), I Targioni Tozzetti fra '700 e '900 (Florence 2006b) 31-39

Vergari, Daniele, «Adolfo Targioni Tozzetti. Il padre della moderna entomologia agraria», in Fausto Barbagli/Daniele Vergari (éds), I Targioni Tozzetti fra '700 e '900, (Florence 2006c) 41-46 